\title{
Inner-Shell-Ionization-Induced Femtosecond Structural Dynamics of Water Molecules Imaged at an X-Ray Free-Electron Laser
}

T. Jahnke $\odot,{ }^{1, *, \dagger}$ R. Guillemin $\odot,{ }^{2, *}$ L. Inhester $\odot,{ }^{3, *,}$ S.-K. Son $\odot,{ }^{3}$ G. Kastirke, ${ }^{4}$ M. Ilchen $\odot,{ }^{1,5}$ J. Rist $\odot,{ }^{4}$ D. Trabert $\odot,{ }^{4}$ N. Melzer $\odot,{ }^{4}$ N. Anders ${ }^{4}$ T. Mazza, ${ }^{1}$ R. Bollø, ${ }^{1}$ A. De Fanis, ${ }^{1}$ V. Music $\odot,{ }^{1,5}$ Th. Weber, ${ }^{6}$ M. Weller, ${ }^{4}$ S. Eckart $\odot,{ }^{4}$ K. Fehre, ${ }^{4}$ S. Grundmann, ${ }^{4}$ A. Hartung, ${ }^{4}$ M. Hofmann, ${ }_{4}^{4}$ C. Janke, ${ }^{4}$ M. Kircher $\odot,{ }^{4}$ G. Nalin $\odot,{ }^{4}$ A. Pier, ${ }^{4}$ J. Siebert, ${ }^{4}$ N. Strenger, ${ }^{4}$ I. Vela-Perez, ${ }^{4}$ T. M. Baumann, ${ }_{1}^{1}$ P. Grychtolø,${ }^{1}$ J. Montano, ${ }^{1}$ Y. Ovcharenko, ${ }^{1}$ N. Rennhack $\odot,{ }^{1}$ D. E. Rivas $\odot,{ }^{1}$ R. Wagner, ${ }^{1}$ P. Ziolkowski, ${ }_{1}^{1}$ P. Schmidt, ${ }^{1,5}$ T. Marchenko $\odot,{ }^{2}$ O. Travnikova $\odot,{ }^{2}$ L. Journel, ${ }^{2}$ I. Ismail $\odot,{ }^{2}$ E. Kukk, ${ }^{7}$ J. Niskanen, ${ }^{7}$

F. Trinter $\odot,{ }^{8,9}$ C. Vozzi $\odot,{ }^{10}$ M. Devetta $\odot,{ }^{10}$ S. Stagira $\odot,{ }^{11}$ M. Gisselbrecht $\odot,{ }^{12}$ A. L. Jäger, ${ }^{13}$ X. Li ${ }_{14}^{14}$ Y. Malakar, ${ }^{14}$ M. Martins $\odot,{ }^{15}$ R. Feifel $\odot,{ }^{16}$ L. Ph. H. Schmidt, ${ }^{4}$ A. Czasch, ${ }^{4}$ G. Sansone, ${ }^{13}$ D. Rolles $\odot,{ }^{14}$ A. Rudenko $\odot,{ }^{14}$ R. Moshammer,${ }^{17}$ R. Dörner, ${ }^{4}$ M. Meyer®, ${ }^{1}$ T. Pfeifer $\odot,{ }^{17}$ M. S. Schöffler, ${ }^{4}$ R. Santra $\odot,{ }^{3,18,8}$ M. Simon $\odot,{ }^{2}$ and M. N. Piancastelli $\odot^{2,19, \|}$

${ }^{1}$ European XFEL, D-22869 Schenefeld, Germany

${ }^{2}$ Sorbonne Université, CNRS, Laboratoire de Chimie Physique-Matière et Rayonnement, LCPMR, F-75005, Paris, France

${ }^{3}$ Center for Free-Electron Laser Science CFEL, Deutsches Elektronen-Synchrotron DESY, D-22607 Hamburg, Germany

${ }^{4}$ Institut für Kernphysik, Goethe-Universität, D- 60438 Frankfurt am Main, Germany

${ }^{5}$ Institut für Physik und CINSaT, Universität Kassel, D-34132 Kassel, Germany

${ }^{6}$ Lawrence Berkeley National Laboratory, Chemical Sciences, Berkeley, California 94720, USA

${ }^{7}$ Department of Physics and Astronomy, University of Turku, FI-20014 Turku, Finland

${ }^{8}$ FS-PETRA-S, Deutsches Elektronen-Synchrotron (DESY), D-22607 Hamburg, Germany

${ }^{9}$ Molecular Physics, Fritz-Haber-Institut der Max-Planck-Gesellschaft, D-14195 Berlin, Germany

${ }^{10}$ IFN-Istituto di Fotonica e Nanotecnologie, CNR, I-20133 Milan, Italy

${ }^{11}$ Politecnico di Milano, Physics Department, Milan, Italy

${ }^{12}$ Department of Physics, Lund University, SE-22100 Lund, Sweden

${ }^{13}$ Physikalisches Institut, Universität Freiburg, D-79104 Freiburg, Germany

${ }^{14}$ J. R. Macdonald Laboratory, Department of Physics, Kansas State University, Manhattan, Kansas 66506, USA

${ }^{15}$ Institut für Experimentalphysik, Universität Hamburg, D-22761 Hamburg, Germany

${ }^{16}$ Department of Physics, University of Gothenburg, SE-412 96 Gothenburg, Sweden

${ }^{17}$ Max-Planck-Institut für Kernphysik, D-69117 Heidelberg, Germany

${ }^{18}$ Department of Physics, Universität Hamburg, D-22607 Hamburg, Germany

${ }^{19}$ Department of Physics and Astronomy, Uppsala University, SE-751 20 Uppsala, Sweden

(Received 25 March 2021; revised 20 July 2021; accepted 16 September 2021; published 3 December 2021)

\begin{abstract}
The ultrafast structural dynamics of water following inner-shell ionization is a crucial issue in high-energy radiation chemistry. We have exposed isolated water molecules to a short $\mathrm{x}$-ray pulse from a free-electron laser and detected momenta of all produced ions in coincidence. By combining experimental results and theoretical modeling, we can image dissociation dynamics of individual molecules in unprecedented detail. We reveal significant molecular structural dynamics in $\mathrm{H}_{2} \mathrm{O}^{2+}$, such as asymmetric deformation and bondangle opening, leading to two-body or three-body fragmentation on a timescale of a few femtoseconds. We thus reconstruct several snapshots of structural dynamics at different time intervals, which highlight dynamical patterns that are relevant as initiating steps of subsequent radiation-damage processes.
\end{abstract}

\footnotetext{
*These authors have contributed equally to this work.

${ }^{\dagger}$ To whom correspondence should be addressed. till.jahnke@xfel.eu

FTo whom correspondence should be addressed. ludger.inhester@desy.de

${ }^{\S}$ To whom correspondence should be addressed. robin.santra@cfel.de

"To whom correspondence should be addressed. maria-novella.piancastelli@physics.uu.se
}

Published by the American Physical Society under the terms of the Creative Commons Attribution 4.0 International license. Further distribution of this work must maintain attribution to the author(s) and the published article's title, journal citation, and DOI. 
DOI: 10.1103/PhysRevX.11.041044

\section{INTRODUCTION}

The response of water to ionizing radiation and the accompanying formation of ions and/or radicals is a key question for many research areas, such as radiation damage in biological tissue [1], radio-oncology [2], and corrosion in nuclear reactors [3]. Moreover, understanding the radiolysis of water is crucial for mechanisms in the magnetosphere of Saturn $[4,5]$ and is essential to explain the formation of $\mathrm{O}_{2}$ in cometary ice [6].

High-energy ionizing radiation can trigger extremely fast processes in water. While several existing techniques can identify the species produced in this way, the exact mechanism and, particularly, the precise timescale of the underlying processes have so far remained elusive. Because of internal relaxation mechanisms, ionization of a core electron in water leads to a valence electron filling the core vacancy and another valence electron being emitted (Auger decay). From gas-phase data, it is known that the resulting water dication $\left(\mathrm{H}_{2} \mathrm{O}^{2+}\right)$ eventually dissociates into fragments, which are mainly either hydroxyl cation $\left(\mathrm{OH}^{+}\right)$or atomic oxygen $(\mathrm{O})$, and protons $\left(\mathrm{H}^{+}\right)$[7]. In a liquid environment, secondary electrons cause further ionization in the surroundings, resulting in valence-ionized water molecules $\left(\mathrm{H}_{2} \mathrm{O}^{+}\right)$. These eventually dissociate via proton transfer, leading to the production of hydroxyl radicals $(\mathrm{OH})$ and hydronium ions $\left(\mathrm{H}_{3} \mathrm{O}^{+}\right)$[3,8-10]. The dynamics of core ionization and core excitation in liquid water have been studied via fluorescence [11,12] and Auger spectroscopy [13]. However, the formation and evolution of ions and radicals upon core ionization, which ultimately determine the subsequent radiation chemistry in the liquid, have remained untraceable.

Probing the dynamics in the liquid is extremely challenging since the dynamics are dominated by the hydrogen motion that has, in general, little impact on spectroscopic features accessible in the femtosecond domain. Assuming that the immediate response to core ionization of water is mostly governed by strong intramolecular forces, insight into the dynamics triggered by core ionization of liquid water can be obtained from gas-phase water. The abundance of ion fragments and their asymptotic momenta produced by core exciting and/or core ionizing an isolated $\mathrm{H}_{2} \mathrm{O}$ molecule have been studied by ion-mass spectroscopy [14] or ion/ion-electron coincidence measurements [7,14]. However, while these techniques allow identification of the produced fragments, they do not shed full light on the temporal evolution of the dissociation events. Studies using intense infrared multiple ionization identify the occurrence of dynamics on a timescale of a few femtoseconds $[15,16]$.
Subject Areas: Atomic and Molecular Physics,

Chemical Physics

Moreover, the dissociation pathway of the $\mathrm{H}_{2} \mathrm{O}^{2+}$ dication has been reported only for the lowest dication states reached after valence double ionization [15-20]. The full characterization of its fragmentation dynamics, including both structural and timing information, and involving the whole ensemble of dicationic states reached after core ionization and Auger decay, is of immediate relevance for the understanding of subsequent radiation chemistry processes.

With the novel opportunities offered by an x-ray freeelectron laser source, in particular, an intensity high enough to allow multiple photon absorption, here we provide a complete picture of the dynamics of the core-ionized water molecule. We demonstrate that, on a timescale of a few femtoseconds after core ionization and subsequent Auger decay, water undergoes structural deformation such as asymmetric $\mathrm{O}-\mathrm{H}$ bond stretching and/or opening of the bond angle all the way up to 180 degrees, eventually leading to rapid two-body or three-body fragmentation in asymmetric and/or unbent geometries. That evolution is traced at different time intervals thanks to a combination of experimental advances and theoretical modeling.

\section{RESULTS AND DISCUSSION}

In Fig. 1, we show the sequence of events most relevant for the purpose of illuminating the dynamics of the coreionized water molecule. Upon absorption of the first photon at time $t_{1}$, core-ionized water is created, and subsequently, a water dication $\left(\mathrm{H}_{2} \mathrm{O}^{2+}\right)$ is formed via Auger decay. Both singly and doubly charged molecular ions undergo structural deformation, where chemical bonds still play a role. Since the dynamics in the core-ionized water are negligible [21], because of the short Auger lifetime (about $3 \mathrm{fs}$ ) and the small effect of the core hole on the nuclear geometry, the relevant fragmentation dynamics occur in $\mathrm{H}_{2} \mathrm{O}^{2+}$ [15-20]. The evolution of the system continues until a second photon is absorbed at time $t_{2}$. After the second core photoionization and the following Auger decay, the total charge is +4 , dissociation induced by strong Coulomb repulsion occurs, and three ions $\left(\mathrm{O}^{2+}, \mathrm{H}^{+}\right.$, $\mathrm{H}^{+}$) are detected in coincidence. The momenta of the three ions preserve information on the structural dynamics undergone in between the absorptions of the two photons. Any deviation from a pure Coulomb explosion in geometries close to the ground-state one is a signature of chemical dynamics in the $\mathrm{H}_{2} \mathrm{O}^{2+}$ species.

In our specific experiment, we exposed water vapor to $\mathrm{x}$-ray pulses provided by the European X-ray Free-Electron 


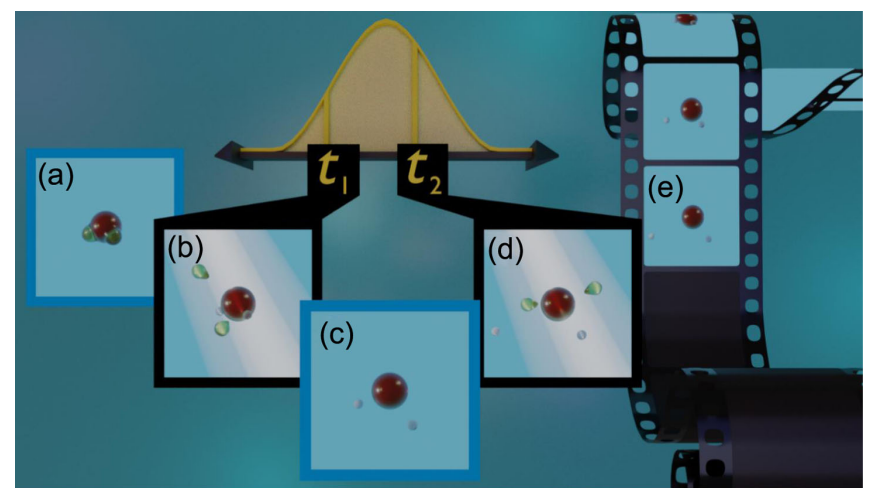

FIG. 1. Two-photon absorption dynamics. (a) Initial $\mathrm{H}_{2} \mathrm{O}$ geometry. (b) As a consequence of the absorption of a photon (visualized using a bright flash) at time $t_{1}$, and subsequent Auger decay, the water molecule loses two electrons (symbolized by the two green blobs ejected). (c) The doubly charged water molecule undergoes structural deformations. The yellow curve on top represents the temporal shape of the photon beam. (d) Through the absorption of a second photon at time $t_{2}$, the molecule becomes quadruply charged and undergoes a Coulomb explosion. The molecular geometry at time $t_{2}$ leads to characteristic momentum vectors for the three fragments, i.e., the two protons and the $\mathrm{O}^{2+}$ ion that are detected for each molecule individually. (e) Using the ion momentum vectors detected in coincidence in combination with theory, information is retrieved on the structural properties of the doubly charged water molecule at a delay of $t_{2}-t_{1}$.

Laser (EuXFEL) at the Small Quantum Systems (SQS) scientific instrument. We employed a cold-target recoil-ion momentum spectroscopy (COLTRIMS) reaction microscope (REMI) a permanent user endstation at SQS to perform triple ion-ion-ion coincidence measurements of the $\mathrm{O}^{\mathrm{n}+} / \mathrm{H}^{+} / \mathrm{H}^{+}$ charged fragments (see the Appendix A for a detailed description of the instrumentation and experimental parameters). The photon energy was fixed at $1 \mathrm{keV}$, high enough to allow consecutive core ionization events [22].

\section{A. Newton diagrams}

The coincidence channel leading to two protons and an oxygen charge $2+$ can be almost entirely associated with sequential absorption of two photons and their subsequent Auger decay process. Figure 2(a) shows the Newton diagrams, i.e., the final momenta of ion fragments $\left(\mathrm{O}^{2+}+2 \mathrm{H}^{+}\right)$detected in coincidence, oriented such that the oxygen momentum points along the $x$ axis. The experimental diagram is compared with simulations of the ionization and fragmentation dynamics conducted with the XMOLECULE toolkit [22-24] (see Appendix A for details on the simulation).

Essentially, the Newton diagrams show two dominant peaks for $\mathrm{H}^{+}$momenta, with a pronounced tail. These main peaks result mostly from an almost instantaneous Coulomb explosion as a result of two photoabsorptions within a very short time interval: The final charge +4 is reached before the dicationic molecular ion has time to undergo a substantial structural rearrangement. In contrast, the pronounced tail in the $\mathrm{H}^{+}$momenta pointing towards the momentum direction of $\mathrm{O}^{2+}$ can be attributed to a scenario where the delay between the two photoabsorption events is sufficiently large, allowing the molecule to evolve after the first photoionization before it is fully ripped apart by the strong Coulomb repulsion induced by the second photoionization. In particular, we underline the role of the kinetic energy release (KER), i.e., the excess energy of the molecular ion that is released as kinetic energy of the fragments. In Fig. 2(b), we show the total KER distribution for the three fragments. In Fig. 2(c), we show the Newton diagrams constructed for different slices of the KER distribution, indicated by different colors in Fig. 2(b), at variance with Fig. 2(a) where the diagrams are shown without KER selection.

One can expect that at larger time delays intermediate dynamics lead, in general, to an increase of interatomic distances, eventually resulting in a lower KER. The trend in the Newton diagrams exhibiting a suppression of the tail with higher KER [see upper and middle panels in Fig. 2(c)], therefore, confirms that the tail corresponds to events with significant time between the two photoabsorptions and thus contains information on the chemical dynamics in $\mathrm{H}_{2} \mathrm{O}^{2+}$, which we are probing. Our approach is an effective pumpprobe method because the time interval between the absorption of two photons, which is the crucial parameter influencing the dynamics, is encoded in the KER (see the discussion about Fig. 4 to further support this assumption). The feasibility of such a method in connection with K-shell photoelectron spectroscopy has recently been demonstrated by some of the authors of this work [25]. A conceptually similar approach has been presented in strong-field experiments, in which a "molecular clock" has been used to monitor the time delay between two photoionization steps occurring within the same pulse [26,27].

\section{B. Direct correlation of proton momenta}

While the Newton diagrams provide a first demonstration of dynamics beyond the Coulomb explosion picture (see Appendix B for further analysis of the tail structure in the Newton diagrams), more detailed insight into the dynamics triggered by core photoionization-Auger events is provided by the scatter plots in Fig. 3. These plots show proton emission angles with respect to the final oxygen momentum direction and the magnitudes of the final momenta of the two protons. All plots are for coincidences with oxygen charge $2+$.

This particular choice of plotting the ion momenta goes beyond the analysis of the usual Newton diagrams and allows us to precisely image specific dynamical patterns since Newton diagrams do not capture all correlations between events. From the correlation of the angles of the two protons shown in Figs. 3(a) and 3(c) 


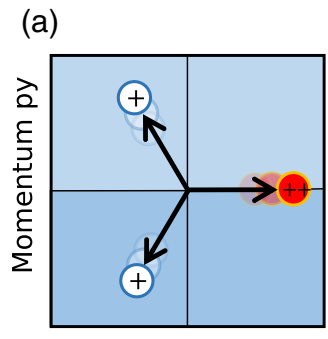

Momentum px

(b)

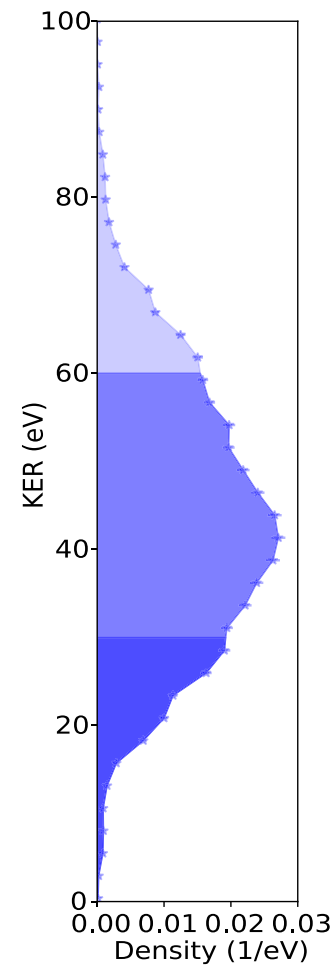

Experiment

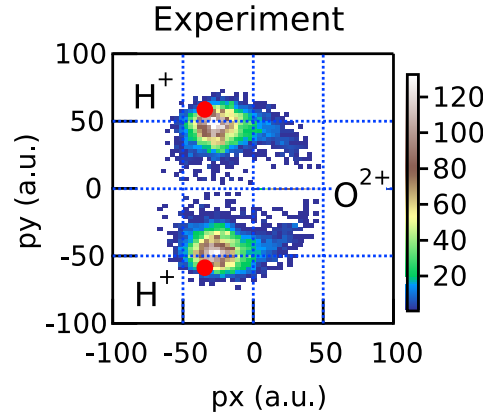

(c)
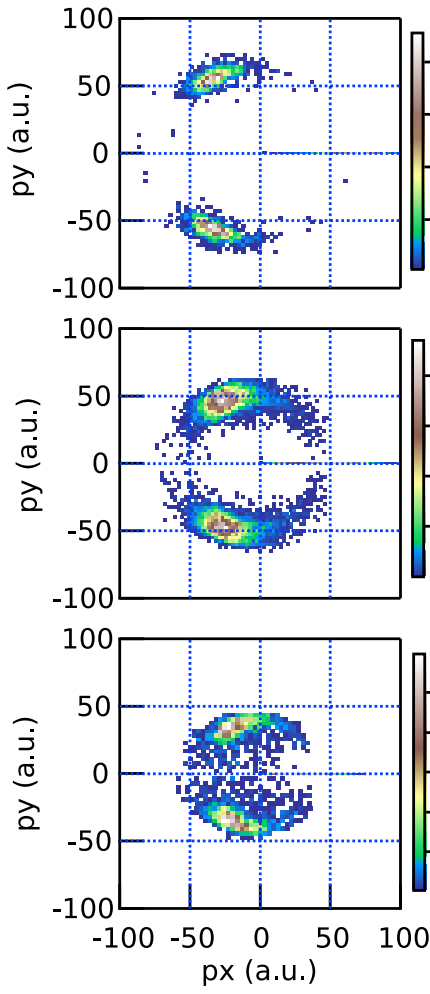
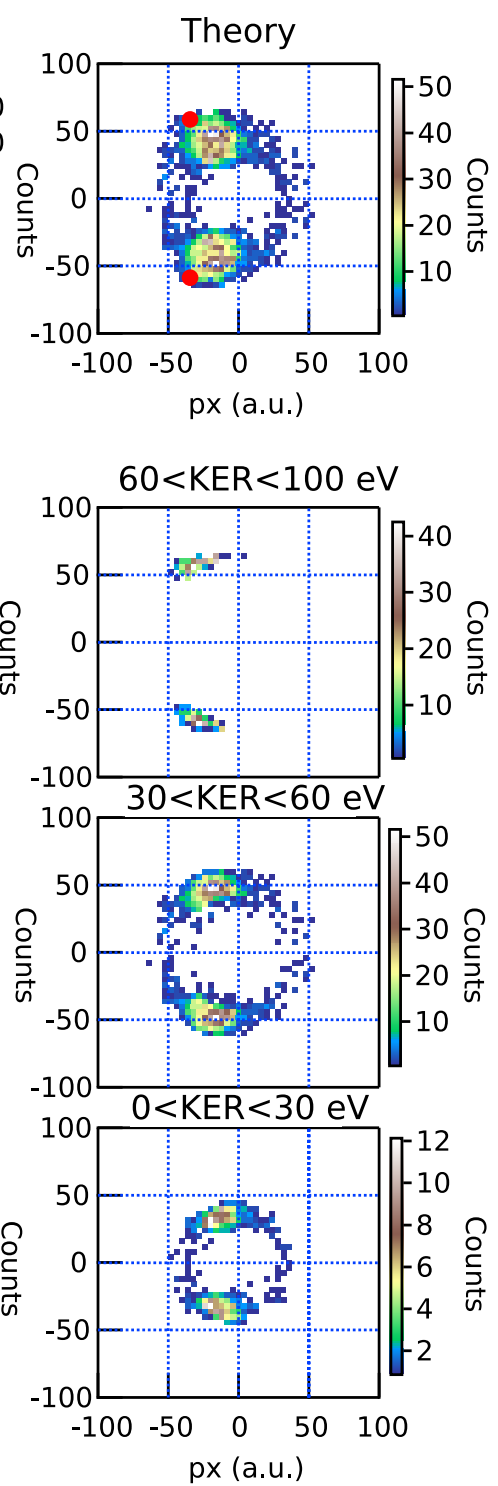

FIG. 2. Newton diagrams. (a) Newton diagrams (experiment and theory) of the ion momenta for $\mathrm{O}^{2+}$ and two $\mathrm{H}^{+}$ions detected in coincidence, where the oxygen momentum defines the $x$ axis, for the full KER range. On the left, the employed momentum coordinate system is shown. The red dots mark the momentum values expected for an instantaneous Coulomb explosion. (b) Distribution of the KER for the three fragments. (c) Newton diagrams (experiment and theory) for selected KER ranges, indicated in (b) by the different color shadings.

(experiment and simulation), it is evident that the main part of the emitted protons is distributed at angles around about $115^{\circ}$, which is close to what one would expect from an immediate Coulomb explosion taking place in almost ground-state geometry. However, more interestingly, in a significant number of events, the angles of the two protons are strongly anticorrelated, and they exhibit an angle sum up to about $180^{\circ}$. In Fig. 3(e) (simulation), the events with large $\mathrm{HOH}$-bond angle at the time of the second photon absorption are shown in green. This signal must be attributed to unbending motion where the water molecule reaches an almost linear geometry at the point in time when the second photoabsorption triggers the final Coulomb explosion.
Figures 3(b) and 3(d) (experiment and simulation) show the absolute momenta of the two protons. Whereas most of the proton momenta are clearly correlated, i.e., they show similar absolute values, a significant fraction of proton momenta is asymmetric. In Fig. 3(f) (simulation), the events in which the two protons have asymmetric momenta at the time of the absorption of the second photon are shown in pink. We can clearly assign these asymmetric proton momenta to asymmetric dissociation that is linked to the dissociation of $\mathrm{H}_{2} \mathrm{O}^{2+}$ into $\mathrm{OH}^{+}$and $\mathrm{H}^{+}$after the first core photoionization and Auger decay. This fragmentation channel of $\mathrm{H}_{2} \mathrm{O}^{2+}$ into $\mathrm{OH}^{+}$and $\mathrm{H}^{+}$is characteristic of the three lowest dicationic states in the water molecule [19,28-30], which accounts for about $25 \%-30 \%$ of the total Auger yield [21,31]. 
(a)

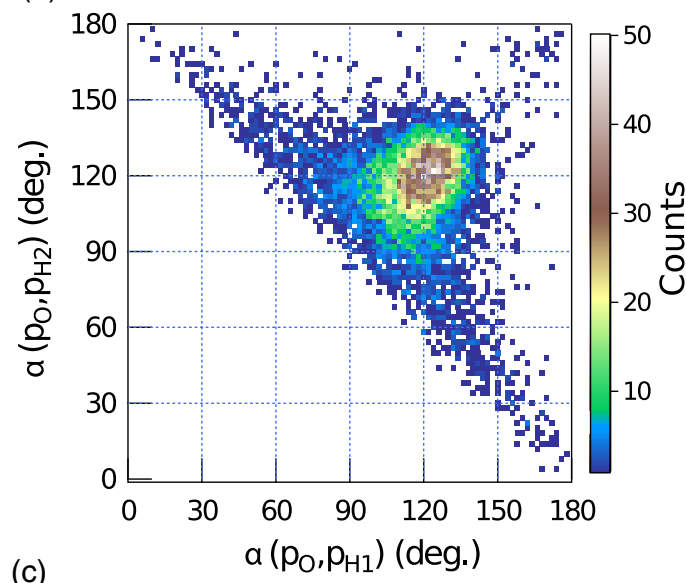

(c)

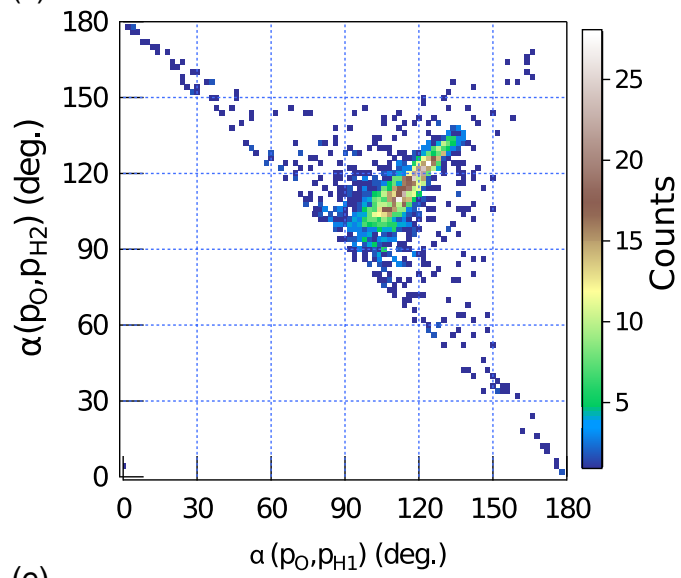

(e)

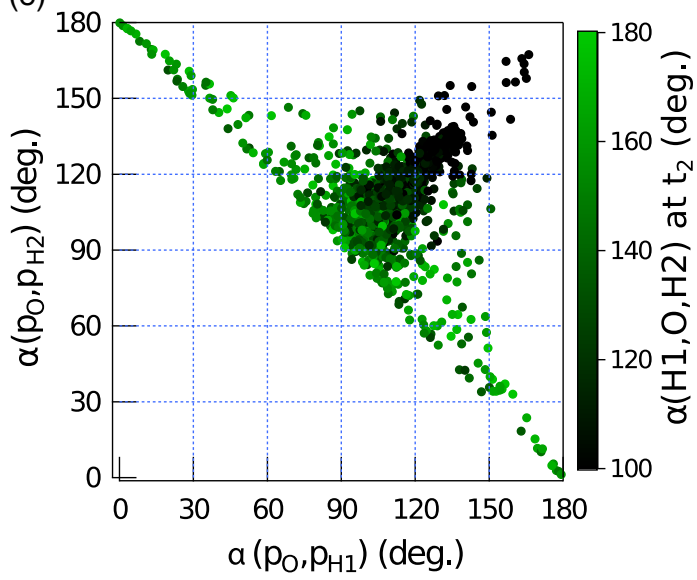

(b)

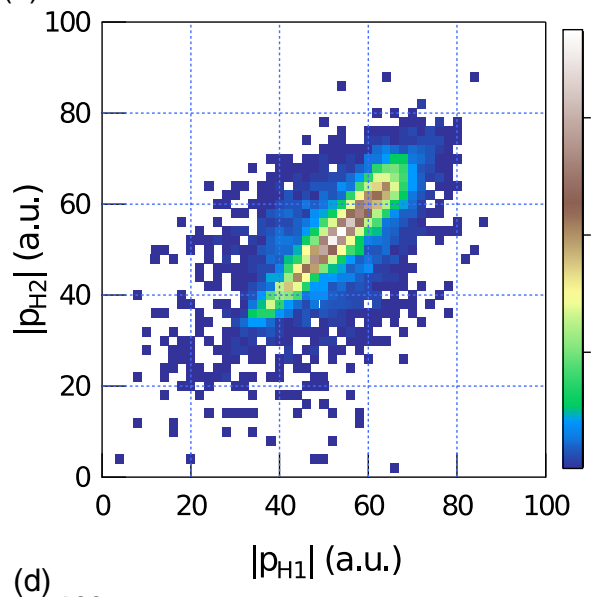

(d)

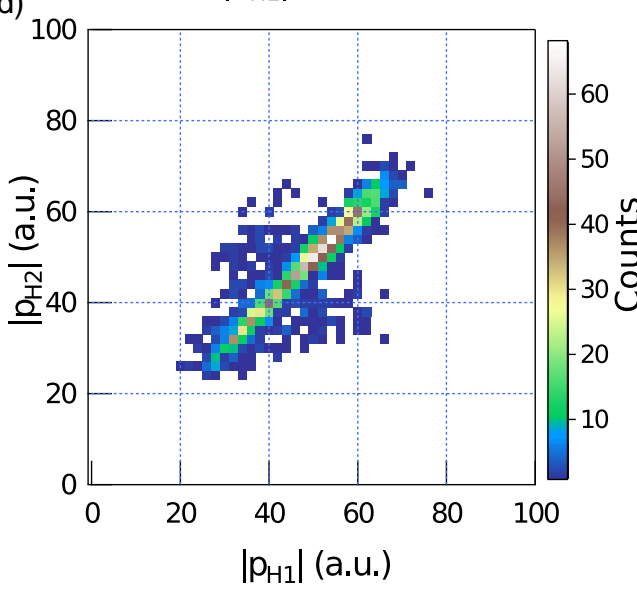

(f)

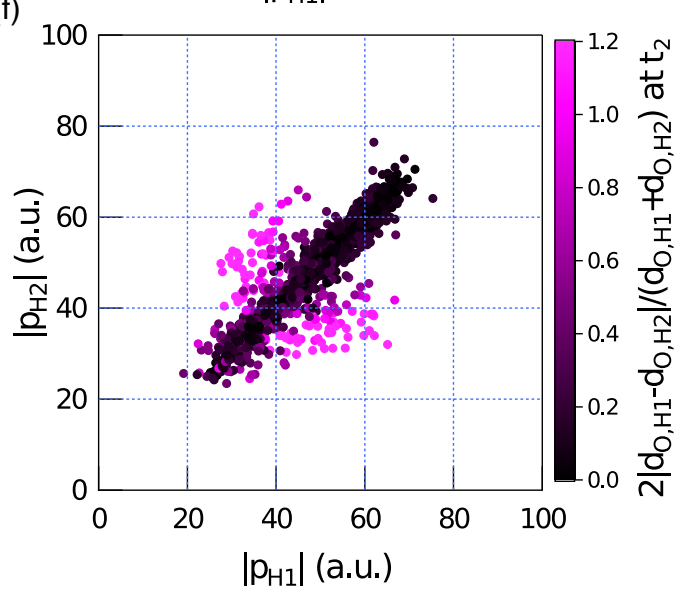

150

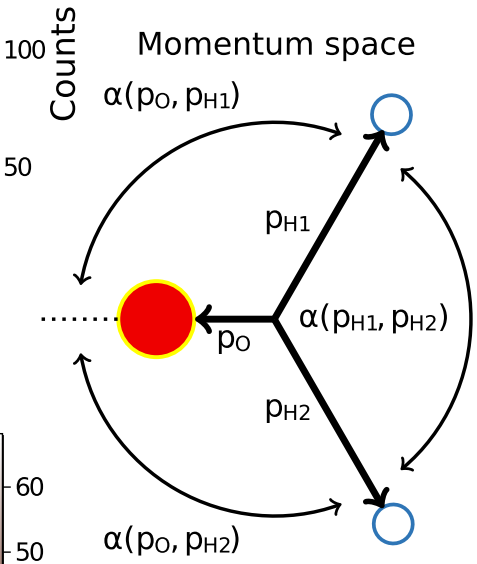

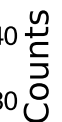

Position space

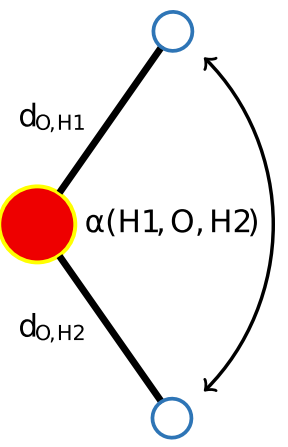

FIG. 3. Scatter plots. (a,c) Scatter plots showing the angles of the final proton momenta with respect to the final oxygen momentum (experiment and simulation). (e) Scatter plot of proton angles (simulation), where a large HOH bond angle at the time of the second photoionization is indicated by green. (b,d) Scatter plots showing the magnitudes of the final proton momenta (experiment and simulation). (f) Scatter plot showing final proton momenta (simulation), where structural asymmetry at the time of the second photoionization is indicated by pink. The relevant momentum- and real-space coordinates are also shown.

\section{Timing information from KER}

The evolution in time of the unbending and asymmetry parameters is reported in Fig. 4. Based on simulation, Fig. 4(a) shows the relation of KER vs time between photoabsorptions (see Appendix $\mathrm{C}$ for a further analysis of the KER on other time parameters). While most of the events correspond to a photoionization-Augerphotoionization-Auger (PAPA) sequence, an alternative path has been identified in previous studies [32], namely, photoionization-photoionization-Auger-Auger (PPAA). PPAA sequences are related to a particularly short time delay between the two photoabsorptions. The two 
(a)

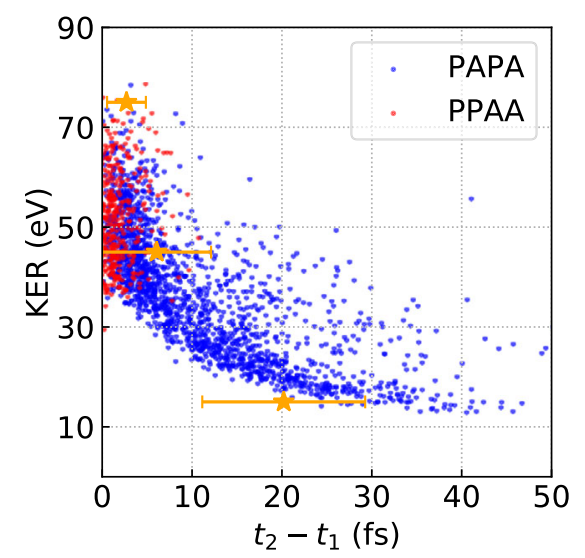

(d)

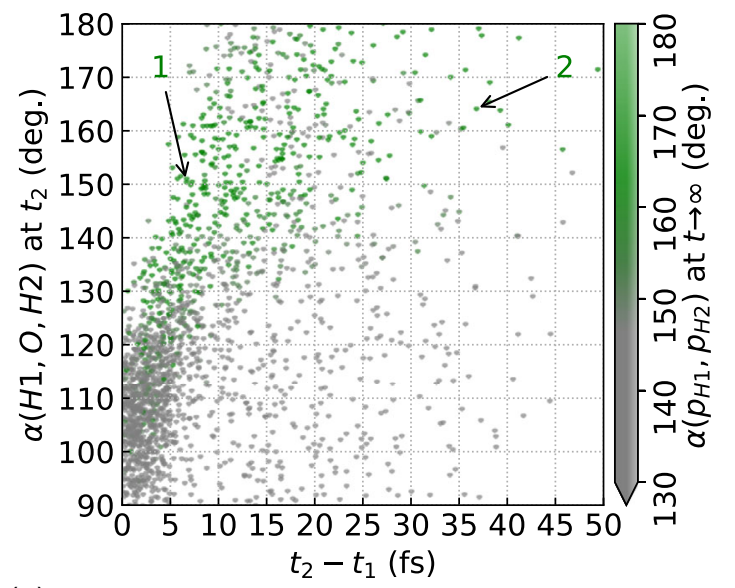

(e)

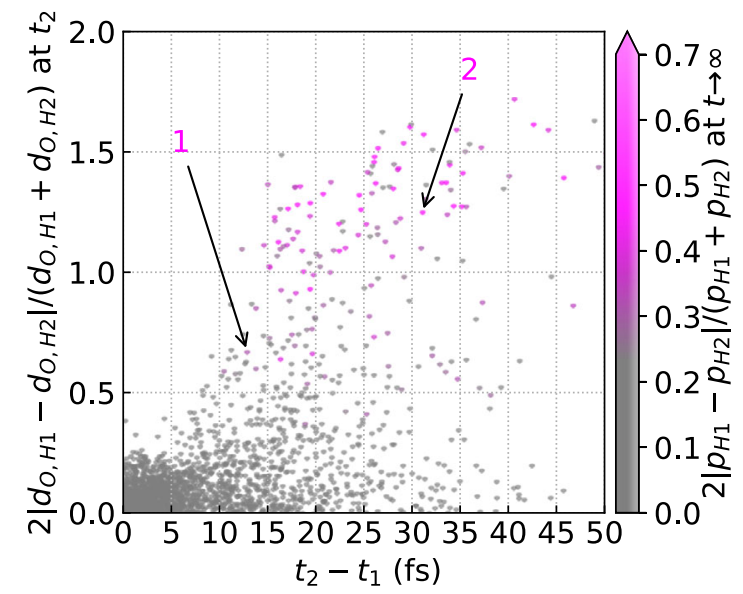

(b) $\quad 20.2 \pm 9 \begin{gathered}t_{2}-t_{1}(\mathrm{fs}) \\ 6.1 \pm 6\end{gathered} \quad 2.7 \pm 2$

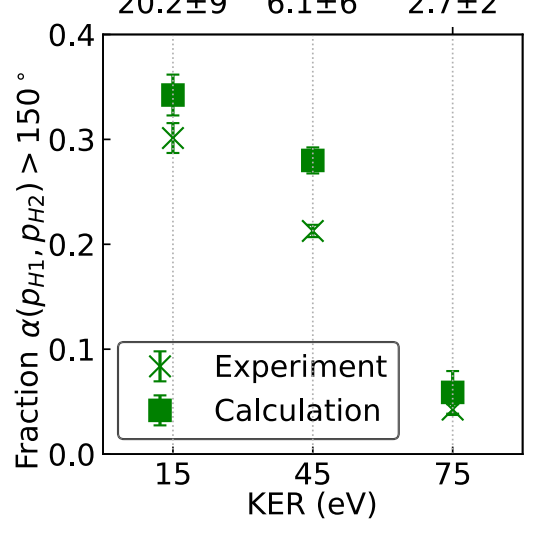

(c)

$20.2 \pm 9 \begin{array}{cc}t_{2}-t_{1}(\mathrm{fs}) \\ 6.1 \pm 6\end{array} \quad 2.7 \pm 2$

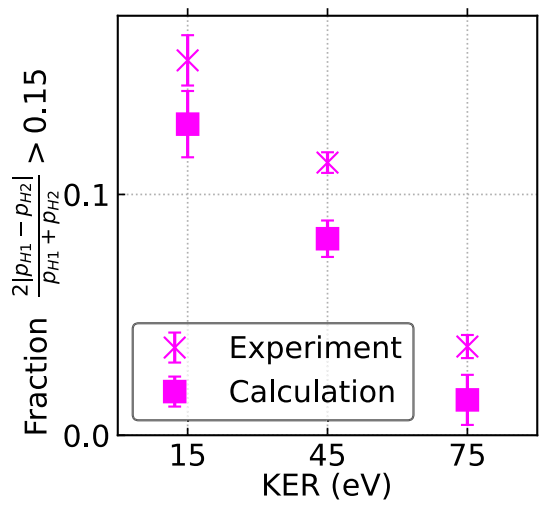

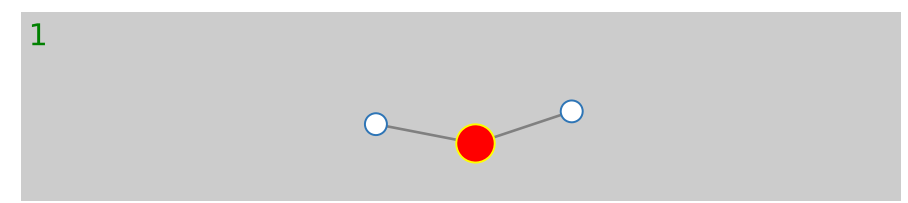

2
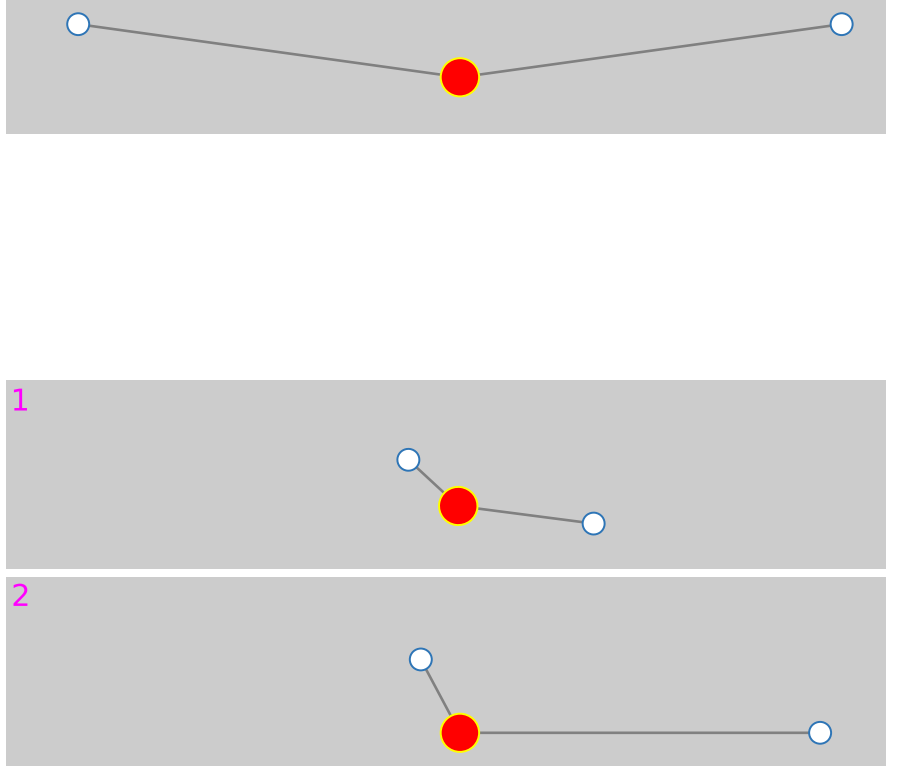

FIG. 4. Evolution in time. (a) KER as a function of time between the first and the second photoionization, $t_{2}-t_{1}$ (simulation). Two different ionization sequences, alternating photoionization and Auger decay (PAPA) and two photoionizations followed by two Auger decays (PPAA), are marked in different colors. The yellow stars mark average delay times for the three KER bins considered in Figs. 1, 4(b), and 4(c). The bars indicate standard deviations of the delay times within the respective KER bins. (b,c) Fraction of coincidence events with large proton-proton momentum angle and large relative proton momentum asymmetry as a function of KER (experiment and simulation). (d,e) $\mathrm{HOH}$ bond angles and asymmetry ratio of the $\mathrm{OH}$ distances at the point in time of the second photoabsorption vs time delay with respect to the first photoabsorption (simulations) accompanied by selected snapshots of the molecular geometry. All plots are for coincidences with oxygen charge +2 and $2 \mathrm{H}^{+}$ions. 
ionization sequences are marked in Fig. 4(a) with a different color code. Figures 4(b) and 4(c) show the fraction of coincidence events with large angle and large relative asymmetry in the proton momenta as a function of KER for the data from both experiment and simulation.

Combining Figs. 4(a)-4(c), lower asymmetry and lower angles, related to higher KER, can, in turn, be connected to shorter times between photoabsorptions. In this way, the increase of bond asymmetry and bond angle is imaged as a function of delay, and snapshots of the dissociating molecular dication are obtained in real time.

The two identified dynamical patterns triggered by the first core-shell ionization and Auger decay-the unbending dynamics and the asymmetric dissociation into $\mathrm{OH}^{+}$and $\mathrm{H}^{+}$- and their consequences for the final fragment momenta can be further understood from the simulation data. Figures 4(d) and 4(e) demonstrate how the bond angle [Fig. 4(d)] and the structural asymmetry [Fig. 4(e)] evolve in time until the second photoabsorption occurs. A large asymmetry in the $\mathrm{OH}$ bond length and a larger $\mathrm{HOH}$ bond angle at this point in time are mapped, respectively, to a large asymmetry and large angles in the final proton momenta. This mapping is reflected by the color labeling, which highlights asymmetry and large angles in the final fragment momenta. The plot also shows that only for sufficient delay times between the two photoabsorption events (about $10 \mathrm{fs}$ and $5 \mathrm{fs}$, respectively), a large asymmetry and/or a large $\mathrm{HOH}$ bond angle is reached (the simultaneous occurrence of the two deformation patterns is possible but not shown for simplicity). We can thus identify the time delay between the two photoabsorption steps as a crucial parameter for the abundance of the observed correlation patterns in the final proton momenta.

\section{Experiment}
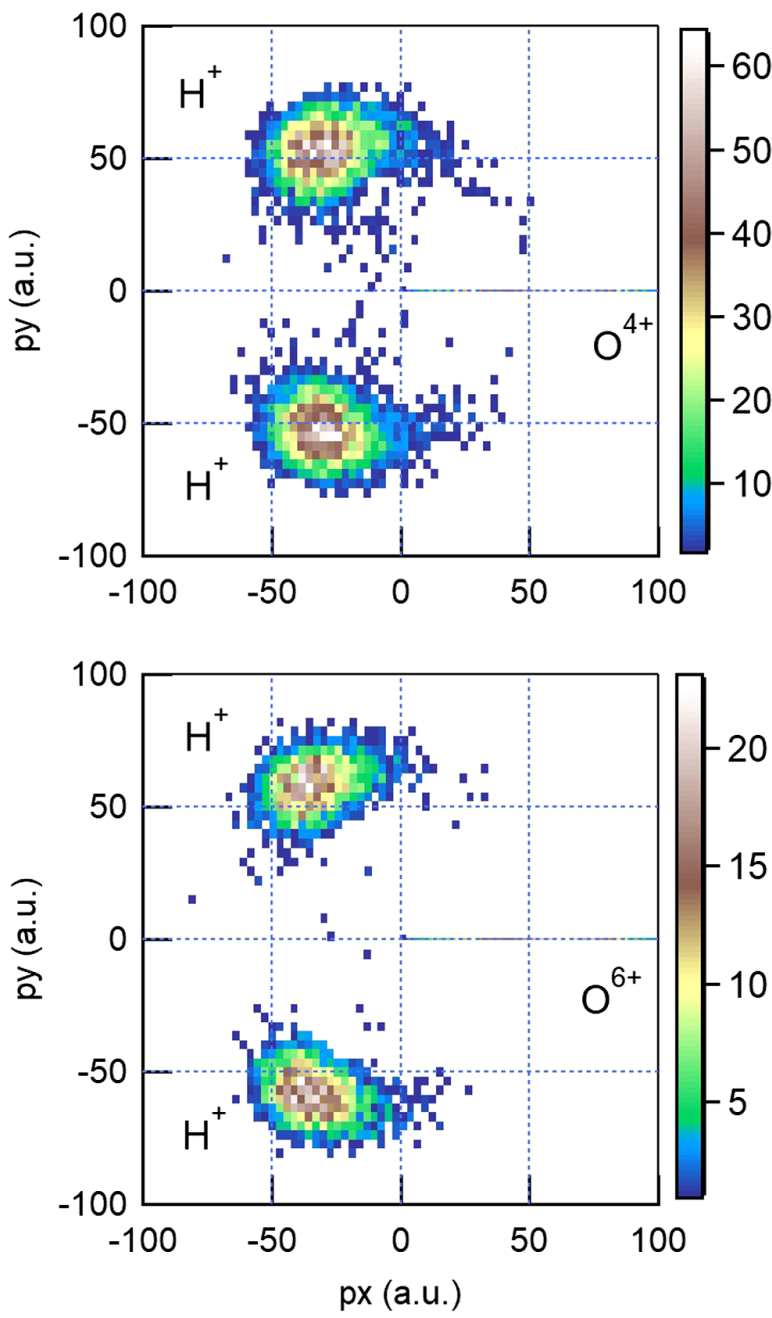

\section{Theory}
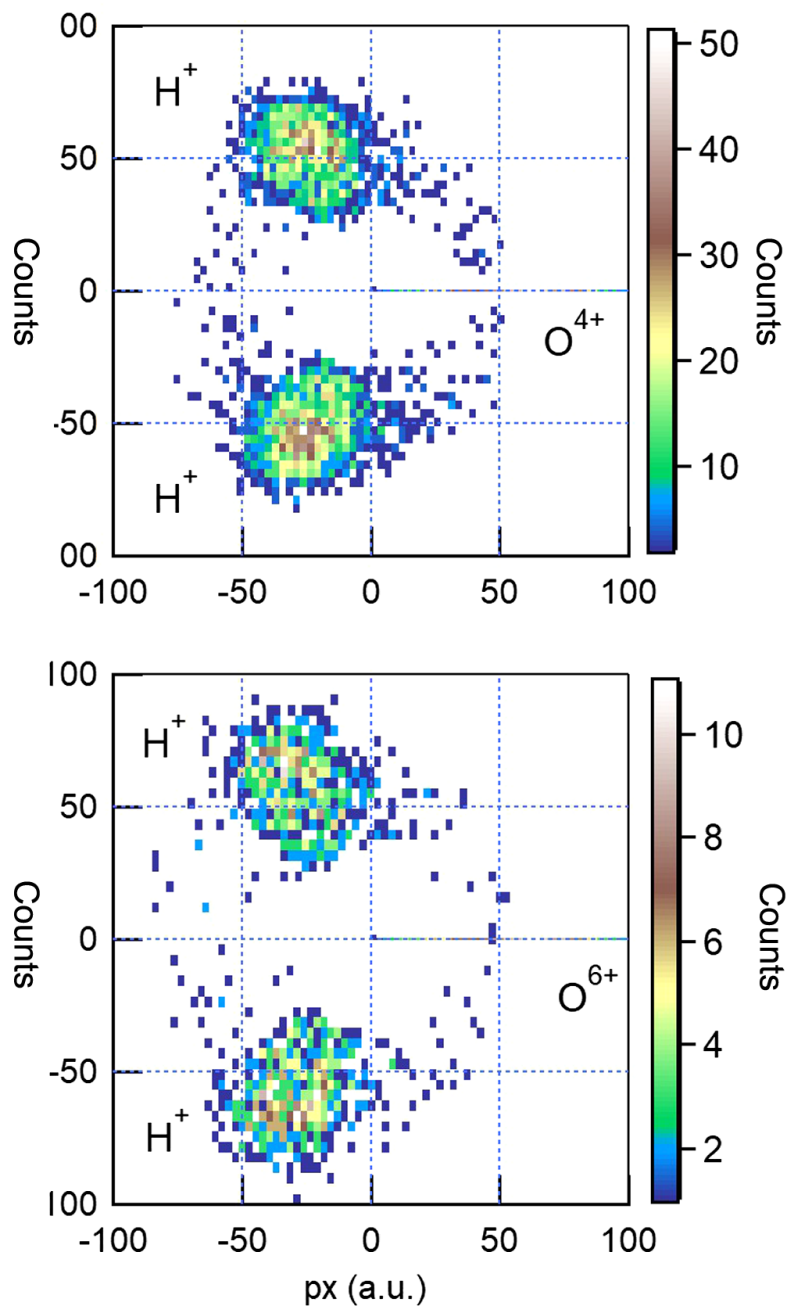

FIG. 5. Newton diagrams for the coincidences $\mathrm{O}^{4+}+2 \mathrm{H}^{+}$and $\mathrm{O}^{6+}+2 \mathrm{H}^{+}$. The left panels show experimental data; the right panels show data from the simulation. 
(a)

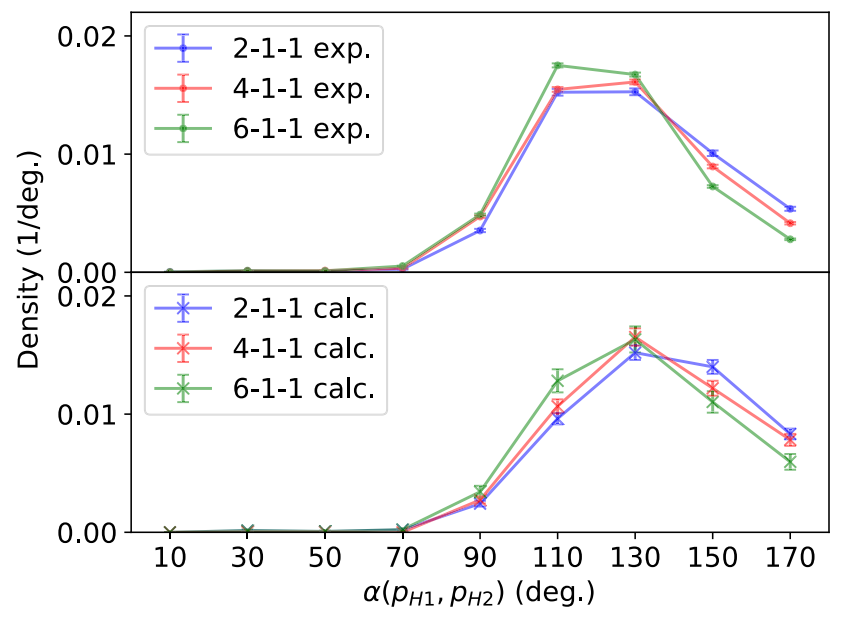

(b)

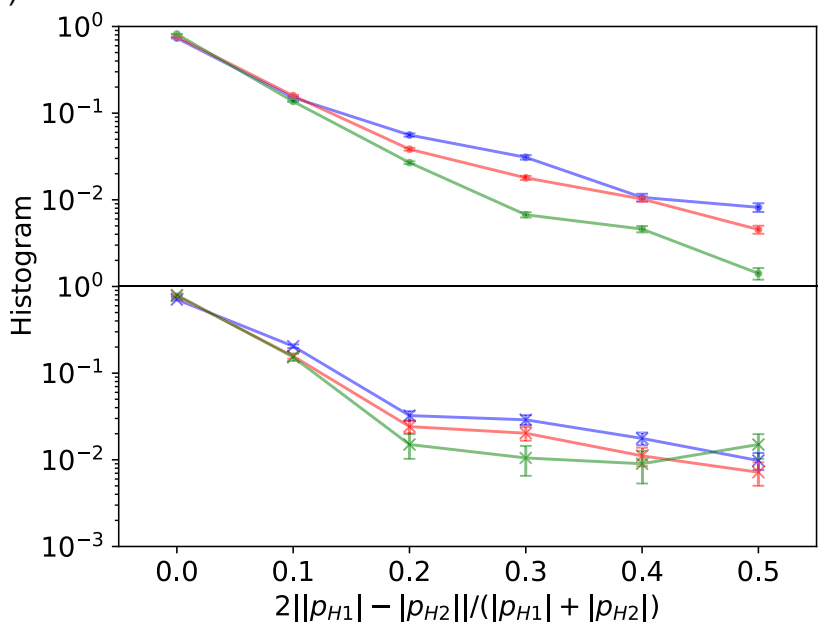

FIG. 6. Distribution of proton momentum angle (a) and proton momentum asymmetry (b) for different charge coincidences (experiment and simulations). The error bars show standard errors due to finite sampling.

The estimated pulse duration at the EuXFEL is about $10-25$ fs. This determines the statistically most probable range of observable delay times, in particular, the time interval between two photoabsorptions. We note that the obtained time resolution is limited by the rather broad distribution of delays $t_{2}-t_{1}$ for a given KER [see Fig. 4(a)]. The employed KER-time relation relies on the mechanism that the bond stretching at the onset of two-body and threebody fragmentation of $\mathrm{H}_{2} \mathrm{O}^{2+}$ results in a universal trend lowering the KER for the quadruply charged molecules. The method to extract time information is, thus, intertwined with the dynamics that we are measuring.

In reality, each dicationic state shows specific deviations from a universal KER $/ t_{2}-t_{1}$ relation that, in combination with the uncertain temporal pulse shape, may result in discrepancies between simulation and experimental data. Moreover, the employed electronic structure model may not accurately cover all details of the involved dicationic potential energy surfaces, which could also cause the observed discrepancies between simulation and experiment in Fig. 4.

\section{Timing information from higher charges}

We strengthen our findings further by making use of coincidences with higher charge states (implying more than two consecutive photoabsorptions) and employing the corresponding timing distribution for these multiple photoabsorptions [33] without relying on a KER-time relation. Figure 5 shows the Newton diagrams for the coincidences with $\mathrm{O}^{4+}+2 \mathrm{H}^{+}$and $\mathrm{O}^{6+}+2 \mathrm{H}^{+}$. As can be seen, the final momentum pattern becomes simpler and more peaked towards a pure Coulomb explosion mechanism as a function of total charge because the time between the first and second photoabsorptions is, on average, shorter when more than two photons are absorbed within the same pulse. The trends for the different coincidences are further quantified in Fig. 6, showing the distribution of proton momentum angle [Fig. 6(a)] and proton momentum asymmetry [Fig. 6(b)] (see Appendix D for the corresponding scatter plots). The two distributions indicate a consistent trend that proton momentum angles larger than $150^{\circ}$ and asymmetries beyond 0.15 are significantly suppressed with higher oxygen charges due to the statistically shorter time in which the molecule evolves in the dicationic state (see Appendix $\mathrm{H}$ for a further discussion on timing information from coincidences at higher charges).

\section{CONCLUSION}

Overall, our study shows that both unbending and twobody fragmentation are significant dynamical features occurring in the fragmentation of $\mathrm{H}_{2} \mathrm{O}$ after core ionization. According to our simulation, about $33 \% \pm 1 \%$ of the formed $\mathrm{H}_{2} \mathrm{O}^{2+}$ molecular ions undergo a two-body fragmentation and about $26 \% \pm 1 \%$ undergo a three-body fragmentation with a proton momentum angle larger than $150^{\circ}$ (see also Supplemental Material, Movies S1-S4 [34]).

Because of their low charge and small electron density, the monitoring of ultrafast hydrogen dynamics is particularly challenging with other imaging techniques such as ultrafast x-ray scattering [35] or ultrafast electron diffraction [36]. The technique employed here, i.e., time-dependent Coulomb-explosion imaging, allows us to separately identify different processes with unprecedented detail. With the short and intense pulses and the high pulse-repetition rate available at the European XFEL, we were able to collect coincident events with sufficient statistics to characterize multiple photoionization processes. This enabled us to unravel dynamical aspects of the radiation-damage 
processes in $\mathrm{H}_{2} \mathrm{O}$. Our experimental findings are in excellent agreement with our computational modeling and simulations of the multiple ionization and fragmentation dynamics.

By combining an appropriate choice of experimental parameters with suitable theoretical modeling, we have exploited the properties of an intense femtosecond $\mathrm{x}$-ray light source to extract dynamical information. In order to characterize more quantitatively the time evolution of a system and to make a so-called molecular movie, which is a sought-after goal, further advances are required. Such advances include better control over the x-ray pulse shape and the application of $\mathrm{x}$-ray pump/x-ray probe experimental setups combined with high repetition rate.

The dynamical patterns we uncovered here have implications for the subsequent radiation chemistry in liquid water. As an example, the momentum of the neutral oxygen is significantly reduced due to the unbending motion, which, in a liquid environment, improves the reactivity of the remaining oxygen atom and might facilitate the formation of $\mathrm{OH}$ radicals.

The immediate response of a water molecule to $\mathrm{x}$ rays and the related molecular deformation and subsequent bond rupture set the stage for radiation chemistry processes, e.g., in a liquid environment. Our insights are therefore broadly relevant in fields related to high-energy radiation damage, not only in the gas phase but also in a multitude of aqueous environments. Our work sets the stage to study further implications of the found dynamical patterns. In the future, these can be accessed by studying small water clusters and extending the accessible timescale by making use of two-Xray pulse setups with variable time delay.

Data recorded for the experiment at the European XFEL are available at [37].

\section{ACKNOWLEDGMENTS}

We acknowledge European XFEL in Schenefeld, Germany, for provision of x-ray free-electron laser beam time at the SQS instrument and would like to thank the staff for their assistance. We are indebted to S. Santra for preparing Fig. 1. This work has been supported by the DOE Office of Science, Office of Basic Energy Sciences, Chemical Sciences, Geosciences, and Biosciences Division grant under Contract No. DE-SC0019451 (L. I., X. L., Y.M., D. R., A.R., and R.S.) and Contract No. DEAC02-05CH11231 (Th. W). M.S., R. G., T.Mar., O. T., L. J., and I. I. acknowledge the financial support of the French CNRS GotoXFEL program. M. I., V. M., and P.S. acknowledge funding by the Volkswagen Foundation within a Peter Paul Ewald fellowship (Volkswagen Foundation, Fund Ref ID, DE/Federal Republic of Germany). This work has been supported by the Bundesministerium für Bildung und Forschung (BMBF), Grants No. 05K13RF4 and No. 05K16RF1. M. S. S., S. G., K. F., T. J., I. V.-P., M. H., and R. D. acknowledge support from Deutsche Forschungsgemeinschaft via
Sonderforschungsbereich 1319 (ELCH). S. E. acknowledges support by the German Research Foundation (DFG) through priority program SPP 1840 QUTIF. F. T. acknowledges support from the MaxWater initiative of the Max-Planck-Gesellschaft. M. D., S. S., and C. V. acknowledge financial support from the Italian Ministry of Research (ELI project-ESFRI Roadmap).

M. N. P. and M. S. originated the project. T. J., M. S. S., G. K., and R.D. devised and built the REMI instrument. Additional help in preparation and commissioning of the REMI and SQS instruments was provided by L. Ph. H. S., A. C., M. W., S. E., K. F., S. G., A. H., M. H., C. J., M. K., G. N., A. P., J. S., N. S., I. V.-P., T. M. B., P. G., J. M., Y. O., N. R., D. E. R., R. W., P. Z., P. S., and M. Me. T. J., M. S. S., M. I., J. R., D. T., N. M., N. A., T. Maz., R. B., A. De F., V. M., and Th. W. executed the experiment and collected the experimental data. T. Mar., O. T., L. J., I. I., E. K., J. N., F. T., C. V., M. D., S. S., M. G., A. L. J., X. L., Y. M., M. Ma., R. F., G. S., D. R., A.R., M. Me., R. M., R. D., T. P., M. S., and M. N. P. participated in the experiment and discussed the results. T. J. and R. G. analyzed the experimental data and interpreted the results. L. I., S-K. S., and R. S. performed the simulations and interpreted the data in terms of dication dynamics. M. N. P., R. S., L. I., S.-K. S., M. S., and R.G. wrote the paper with input from all authors.

\section{APPENDIX A: METHODS}

\section{SQS photon source}

The experiment was performed at the European XFEL in Schenefeld, Germany [38], at the SQS scientific instrument [39]. The European XFEL provides femtosecond x-ray pulses in trains at a repetition rate of $10 \mathrm{~Hz}$. In the present experiment, the pulse spacing within the trains was 1.1 $\mathrm{MHz}$, from which we employed every third to fourth bunch with up to a total of 97 pulses per train and, therefore, an effective number of 970 pulses per second, depending on the required time window for detecting the ions in the reaction microscope. Using a bunch charge of $250 \mathrm{pC}$ at $14-$ $\mathrm{GeV}$ electron energy in the accelerator, the SASE3 soft $\mathrm{x}-$ ray undulators delivered $\mathrm{x}$-ray pulses with durations of about 10-25 fs. The pulse duration was estimated by simulations and indirectly deduced from spectral measurements. Pulse energies of $4.4 \mathrm{~mJ}$ with a typical shot-to-shot variation on the order of $10 \%$ were achieved. The photon energy bandwidth was determined to be about $0.9 \%$. The SQS beamline transmission for the photon energy of $1 \mathrm{keV}$ was measured to be $45 \%$. The focus in the interaction volume was produced by a pair of highly polished mirrors mounted in Kirkpatrick-Baez geometry and was determined to be as small as $1.6 \times 1.4 \mu \mathrm{m}^{2}$ via a Hartmann-type wavefront sensor. By our simulations, we estimated that the average focus during the experiment was, however, somewhat larger, i.e., between $2.0 \times 2.0 \mu \mathrm{m}^{2}$ and $2.9 \times 2.9 \mu \mathrm{m}^{2}$. Further details on how we estimated the fluence distribution of the pulse can be found in Appendix E. 


\section{COLTRIMS reaction microscope}

The experimental data presented in this article were measured using a COLTRIMS reaction microscope [40], which is available through the SQS instrument of the EuXFEL. In our measurement, a supersonic gas jet consisting of a mixture of water vapor and helium was intersected with the x-ray beam of the EuXFEL. Ionic reaction fragments generated by the ionizing $\mathrm{x}$ rays were guided by an electric field to a large area (120-mm diameter) microchannel plate detector with delay-line position readout [41]. The impact times $t$ and the positions of impact $(x, y)$ of all ionic fragments were recorded by means of fast analog-to-digital converters. All ions from a single photoreaction were measured in coincidence. From the $(x, y, t)$ information, the ions' trajectories inside the COLTRIMS spectrometer were reconstructed. From these, the ions' initial vector momenta were deduced. The electric field at the target region was set to $E=213 \mathrm{~V} / \mathrm{cm}$, and the total distance from the target region to the detector was $250 \mathrm{~mm}$. In order to limit the overall voltage, which needed to be applied to the spectrometer and the detector to a feasible amount of $\pm 4000 \mathrm{~V}$, the high acceleration field of $213 \mathrm{~V} / \mathrm{cm}$ was limited to a region of $153 \mathrm{~mm}$. The remaining part of the spectrometer was set to the same voltage as the exit of the acceleration region. This unusual compromise yielded an electrostatic lens that slightly degraded the ion momentum resolution.

The gas target was generated by expanding a mixture of water vapor and helium through a nozzle with a diameter of $200 \mu \mathrm{m}$. The water reservoir, gas line, and nozzle were heated to $40^{\circ} \mathrm{C}, 50^{\circ} \mathrm{C}$, and $70^{\circ} \mathrm{C}$, respectively. The supersonic gas jet was formed by using a three-stage skimmer setup and a set of adjustable slits in front of the last skimmer.

\section{Experimental data analysis}

Multicoincidence experiments on molecular fragments may suffer from false coincidences, e.g., detected fragments that do not belong to the same parent molecule. This is true, in particular, if extreme radiation such as XFEL light is employed for ionization. Typically, the ion detection efficiency and the average ion count rate per XFEL pulse provide a first estimate of whether the data are strongly affected by such false coincidence events. In the present experiment, the ion detection efficiency is in the range of $40 \%$ to $60 \%$. The average number of ions per shot was adjusted using collimating apertures in the molecular beam, yielding approximately 1.3 ions/shot for the lowest fluence measurements and 4.1 ions/shot at the highest XFEL fluence. In our experimental approach, however, the amount of false coincidence events can be drastically reduced by examining (and restricting) the sum of the momenta of all measured ions in the data analysis. In the analysis, the sum of all measured ions is examined. After subtracting momenta to consider the average linear momentum of the absorbed photons and the average molecular beam velocity, this sum momentum depicts the recoil momentum of the emitted electrons. Therefore, we considered events as true coincidences if the sum momentum is less than 20 a.u. Restricting the data set to these events, we estimated a contamination of the data with false coincidence events (for the highest XFEL fluence) by 7\%. A particular case is the break-up channel yielding $\mathrm{D}^{+} / \mathrm{D}^{+} / \mathrm{O}^{4+}$. As our measurement was sensitive to the $\mathrm{m} / \mathrm{q}$ ratio of the ions and not to $\mathrm{m}$ or $\mathrm{q}$ individually, this channel was contaminated by $\mathrm{He}^{+}$ions (from our carrier gas) measured in coincidence with two $\mathrm{D}^{+}$ions (as the $\mathrm{m} / \mathrm{q}$ ratio of $\mathrm{He}^{+}$and $\mathrm{O}^{4+}$ is the same). These contaminations differed, however, in KER, as indicated in Fig. 9.

Molecules in the gas phase are randomly oriented in space. The measured coincidence data can, however, be transferred to a molecular coordinate frame by selecting the emission direction of ions as a reference. For the molecularframe momentum distributions depicted in Fig. 2, the Newton diagrams, the $x$ axis of the coordinate frame was defined to point along the measured momentum of the oxygen ion. The momenta of the proton ions were used to define the $y$ axis, the direction of which is defined such that the $y$ component of the first-arriving proton momentum is always positive and the second is always negative. Momentum analysis also allows us to deduce the angle between ions, as depicted in Fig. 3 in the main text.

\section{Simulations}

Under the intense $\mathrm{x}$-ray pulse, a molecule undergoes several ionization processes. X-ray light preferably ionizes core electrons, and the created holes are predominantly refilled via Auger decay. Because of the high intensity, photoionization can happen many times such that, ultimately, the water molecule can be stripped off all electrons. For the water molecule, this means that many hundreds of different electronic configurations can be populated in the course of the ionization dynamics. The described dynamics is a challenge for electronic structure calculations and demands an efficient electronic structure model capable of handling all the involved highly excited and multiply ionized electronic configurations. To model the many ionization steps and the accompanying fragmentation dynamics, we employed the XMOLECULE electronic structure toolkit $[22,23]$. Within XMOLECULE, we solved the electronic structure of the water molecule for any given electronic configuration. Here, we employed the HartreeFock (HF) electronic structure model in combination with the $6-31 \mathrm{G}(\mathrm{d}, \mathrm{p})$ basis set [42]. For open-shell configurations, we applied the restricted open-shell Hartree-Fock variant for the respective high-spin configuration. For each electronic configuration, photoionization cross sections, Auger decay rates, and fluorescence rates were calculated [22]. The ionization and fragmentation dynamics were simulated by employing the kinetic Monte Carlo technique, 


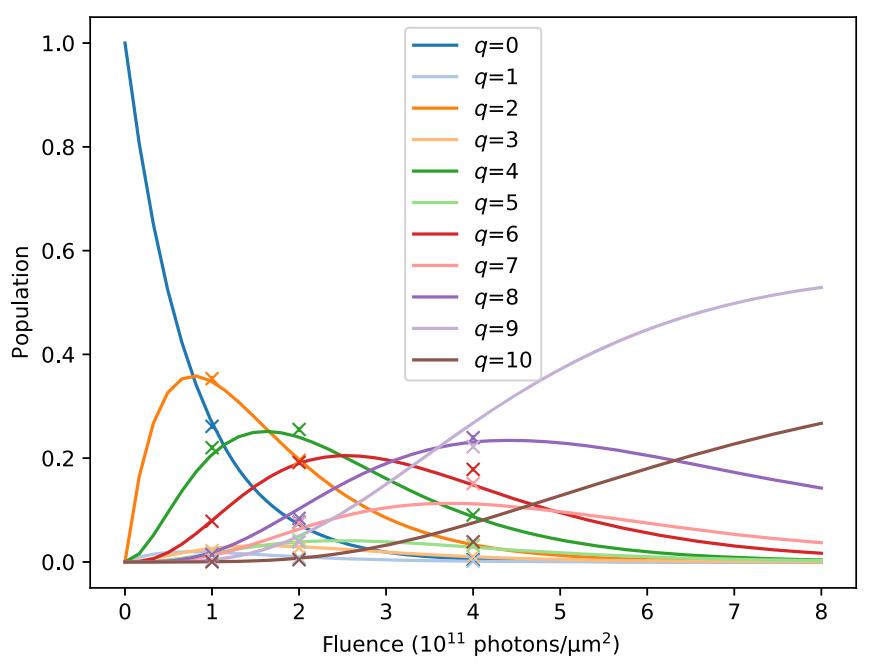

FIG. 7. Population of different charge states $q$ as a function of fluence. The solid lines show results for a fixed-geometry simulation; the crosses show results for a dynamic geometry calculation.

where we propagated the nuclei with forces calculated on the fly, and in each time step, we randomly conducted electronic transitions (photoionization, Auger decay, fluorescence) according to calculated probabilities [43]. The simulation of electronic dynamics is similar to previous works $[22,24]$ where the nuclei have been fixed.

For the current simulation, we used a photon energy of $1 \mathrm{keV}$. The starting geometry and momenta were sampled from a Wigner distribution of the vibrational ground state. For the molecular dynamics, we used a time step of $0.1 \mathrm{fs}$ and propagated each trajectory for a total time of $200 \mathrm{fs}$, where the trajectories began 90 fs before the x-ray pulse's peak. For each parameter set, we collected 8000 trajectories, respectively. To verify our methodology, we also conducted simulations for the deuterated and semideuterated water molecule $\left(\mathrm{D}_{2} \mathrm{O}\right.$ and $\left.\mathrm{HDO}\right)$.

Since the x-ray pulse has an inhomogeneous spatial fluence distribution, different molecules in the sample beam are exposed to different fluence values of up to $8 \times 10^{11} \mathrm{ph} / \mu \mathrm{m}^{2}$ (see Appendix $\mathrm{E}$ for details on the fluence distribution of the pulse). The number of absorbed photons and, consequently, the yield for a specific charge state depend on the fluence. This fluence dependence is illustrated in Fig. 7, showing the population of charge states as a function of fluence. As can be seen, the highest yield for quadruply charged states is achieved at fluences around $2 \times 10^{11} \mathrm{ph} / \mu \mathrm{m}^{2}$, whereas at larger fluences, the population of quadruply charged molecules drops and is transferred to higher charge states.

For the fluence value of $2 \times 10^{11} \mathrm{ph} / \mu \mathrm{m}^{2}$, Fig. 8(a) shows the number of trajectories for different numbers of absorbed photons. As can be seen, with the employed fluence value, we cover a broad region of ionization dynamics ranging from zero-photon absorption (no ionization at all) to up to six absorbed photons, where one-, two-, three-, and four-photon absorptions dominate. In Fig. 8(b), one can see the number of trajectories for the different fragmentation channels, where $q_{O}-q_{\mathrm{H} 1}-q_{\mathrm{H} 2}$ labels the charges of the respective atomic fragments after three-body fragmentation. In most cases, the absorption of a photon leads to ionization of the core shell that most likely induces a further ionization step via Auger decay. This leads to the alternating sequence in the abundance of fragment charges: even charge states (0-1-1, 2-1-1, and 4-1-1) are more abundant than odd charge states (1-1-1, 3-1-1, 5-1-1)
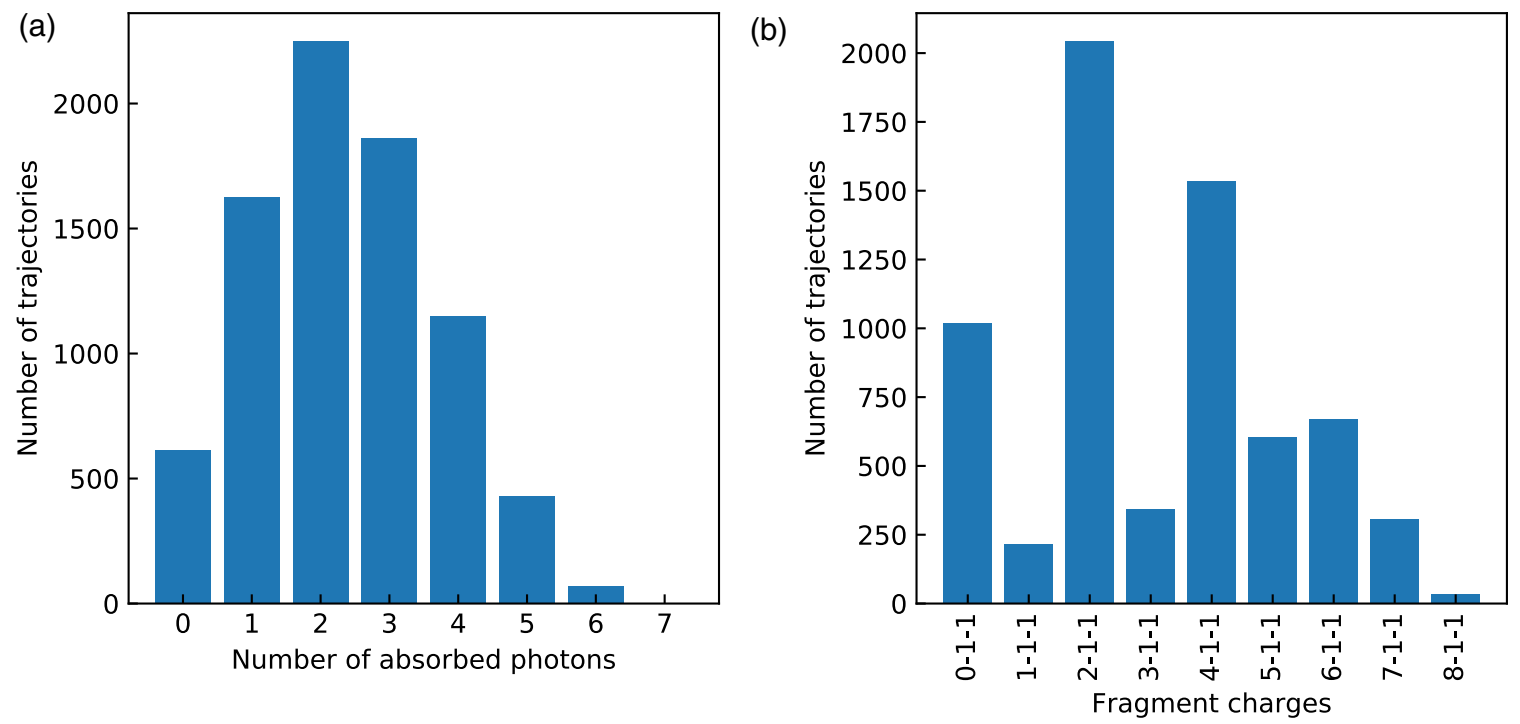

FIG. 8. Number of trajectories for $\mathrm{H}_{2} \mathrm{O}$ grouped according to the number of absorbed photons (a) and the fragment charges (only three-body fragmentations) (b). The total number of trajectories is 8000 . 

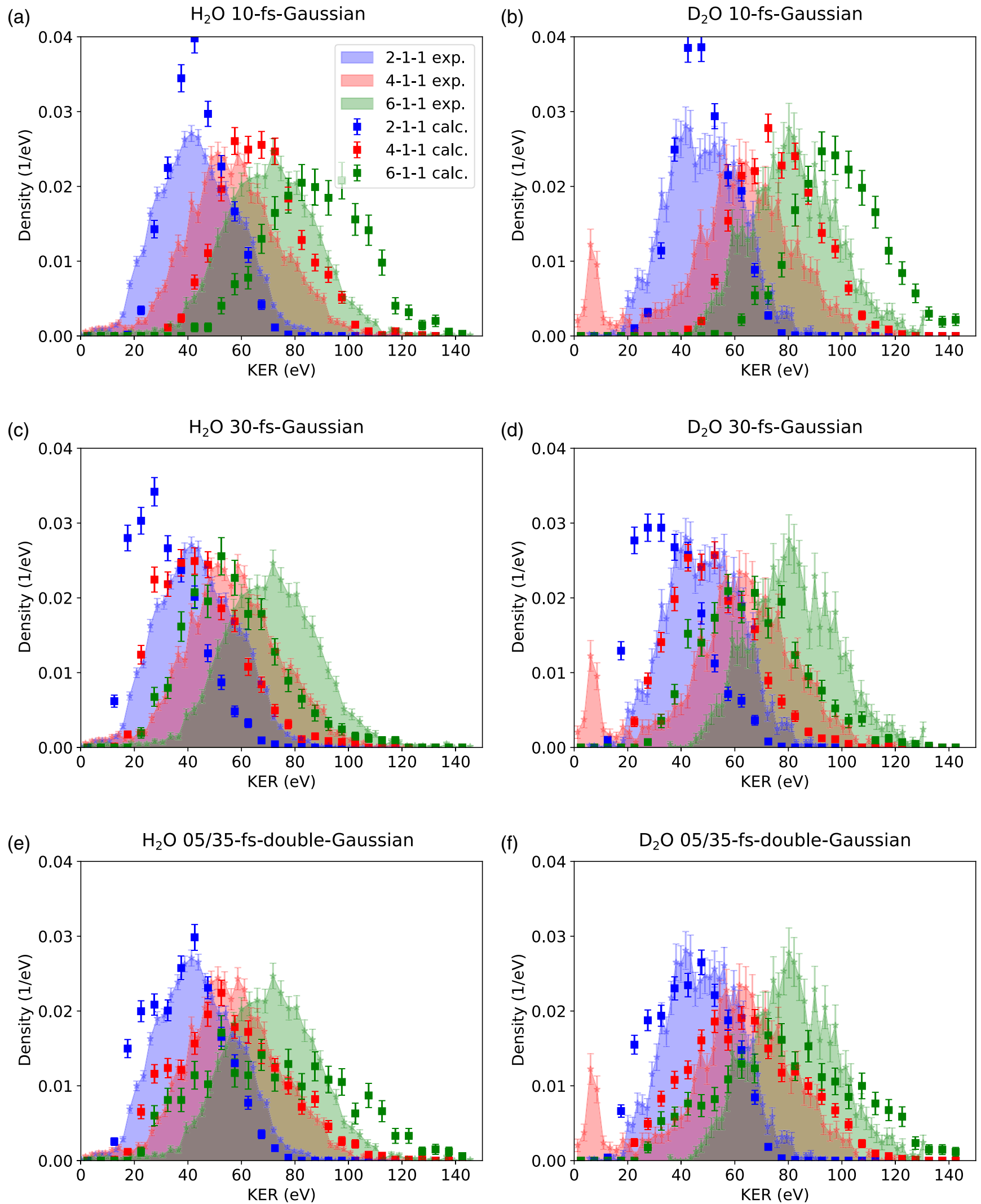

FIG. 9. Total kinetic energy release for $\mathrm{H}_{2} \mathrm{O}$ and $\mathrm{D}_{2} \mathrm{O}$ for different coincidence channels and for different temporal pulse shapes. Here, $\mathrm{x}-1-1$ labels coincidence channels with final oxygen fragment charge $+\mathrm{x}$ and hydrogen charges +1 . The squares show data from the simulation; the asterisks and the shaded areas show data from the experiment. The error bars show standard errors due to finite sampling. Note that the peak at $10 \mathrm{eV}$ for $\mathrm{D}_{2} \mathrm{O}$ in the experimental data is an artifact caused by $\mathrm{He}^{+}$. 
[22,24]. Accordingly, 0-1-1, 2-1-1, 4-1-1, and 6-1-1 can be almost exclusively identified with $1,2,3$, and 4 core-shell photoionizations, respectively. We do not see any neutral hydrogen fragments.

Because of the clear association of charge states with the number of absorbed photons, the fragmentation dynamics within a given charge coincidence varies only slightly with the fluence in the interaction region. (See a detailed discussion on this in Appendix F.) If not mentioned otherwise, our analysis is based on a fluence value of $F=$ $2 \times 10^{11} \mathrm{ph} / \mu \mathrm{m}^{2}$ because it yields a large amount of the charge state $q=+4$.

\section{a. Temporal pulse shape}

In our simulations, we observed that details of the fragments' final momenta sensitively depend on the actual temporal pulse shape. Since the precise shape of the x-ray pulse envelope in the experiment is unknown, we used a number of different pulse shapes to model the resulting fragmentation: a Gaussian with a full width at half maximum (FWHM) of 10 fs, a Gaussian with a FWHM of $30 \mathrm{fs}$, and a double Gaussian,

$$
f(t)=F\left(0.6 f_{5 \mathrm{fs}}(t)+0.4 f_{35 \text { fs }}(t)\right),
$$

where $f_{5 \text { fs }}(t)$ and $f_{35 \text { fs }}(t)$ are Gaussian functions with a FWHM of 5 fs and $35 \mathrm{fs}$, respectively.

For three different temporal pulse shapes, Fig. 9 compares the resulting KER for different coincidence channels and for $\mathrm{H}_{2} \mathrm{O}$ and $\mathrm{D}_{2} \mathrm{O}$ that have been measured in the same experiment. As can be seen, the 10-fs FWHM Gaussian pulse [Figs. 9(a) and 9(b)] leads to too-large KER compared with the experimental data for the charge coincidences 4-1-1 and 6-1-1. In addition, for the 2-1-1 coincidences, the distribution is somewhat too narrow. The 30-fs FWHM Gaussian pulse [Figs. 9(c) and 9(d)] yields too-low KER for all coincidences. Overall, we see a somewhat better agreement between experimental and calculated KER for the 5-fs/35-fs double-Gaussian pulse shape [Figs. 9(e) and 9(f)], where the KERs of the simulations are only slightly lower than the experimental values.

The reported trends are consistent for $\mathrm{D}_{2} \mathrm{O}$ and $\mathrm{H}_{2} \mathrm{O}$ and therefore suggest that the remaining mismatch is largely not due to a discrepancy in the involved potential energy surfaces but rather due to further details that are missing in the temporal pulse shape employed in the calculation. If not stated otherwise, the presented results are shown for the double-Gaussian temporal pulse shape, as it shows the best agreement with the experimental data. For an extended discussion on how different temporal pulse shapes impact the extraction of time information, see Appendix G.

\section{b. Dicationic fragmentation}

For the trajectories with single photoabsorption, we observe different fragmentation channels. The majority of trajectories shows a three-body breakup into $\mathrm{O}+$ $2 \mathrm{H}^{+} / \mathrm{D}^{+}$, while a smaller part $(33 \pm 1 \%, 34 \pm 1 \%, 30 \pm$ $1 \%$ for $\mathrm{H}_{2} \mathrm{O}, \mathrm{D}_{2} \mathrm{O}$, and $\mathrm{HDO}$, respectively) shows twobody breakup into $\mathrm{OH}^{+} / \mathrm{OD}^{+}+\mathrm{D}^{+} / \mathrm{H}^{+}$. We see a strong asymmetry regarding the $\mathrm{OH}^{+}$and $\mathrm{OD}^{+}$production from $\mathrm{HDO}$, resulting in $\mathrm{OD}^{+}$being about 5 times more probable than $\mathrm{OH}^{+}$. Both of these observations are in excellent agreement with earlier findings $[18,44,45]$ and thus confirm the accuracy of our theoretical model.

\section{c. Double core holes}

For the trajectories resulting in a total charge +4 , which are almost exclusively due to two core-shell photoionization and two Auger decay events, we observe several events where the two photoionizations happen so rapidly that the Auger decay has not yet refilled the initial core vacancy. The created double core hole is subsequently refilled via two Auger decay events. For the fluence value of $F=2 \times 10^{11} \mathrm{ph} / \mu \mathrm{m}^{2}$, the ratio of double-core-hole creation (PPAA sequence) is $17 \%$, whereas $82 \%$ of the trajectories exhibit a sequence where photoionization is directly followed by Auger decay (PAPA sequence). Whereas PPAA sequences result in ion fragments with particularly high KER, consistently with the required short delay time $t_{2}-t_{1}$ [see Fig. 4(a)], our simulation data do not exhibit any feature in the ion momentum data that allows us to uniquely distinguish PPAA from PAPA events. Notably, such a distinction would be possible with coincidently detected photoelectrons due to the distinct photoelectron lines of the two sequences [24].

\section{APPENDIX B: ANALYSIS OF THE TAIL STRUCTURE IN THE NEWTON DIAGRAMS}

In the Newton diagrams, a considerable part of the protons forms a tail pointing towards the momentum of the oxygen. This tail structure cannot solely be associated with a sequential fragmentation (as has been done in different contexts before [46-49]).

To elucidate the relation of the tail structure in the Newton diagrams in Fig. 2 with dynamics in the dication, we show in Fig. 10(a) the final momentum data from the simulation in a Newton diagram (the oxygen momentum direction defines the $x$ axis), where we have now marked 
(a)

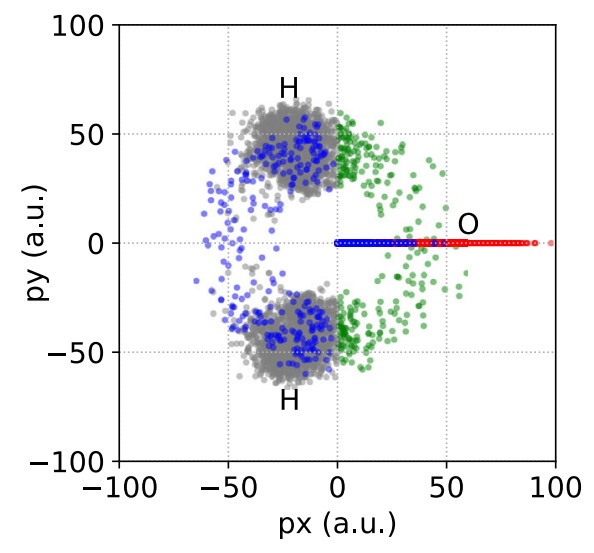

(b)

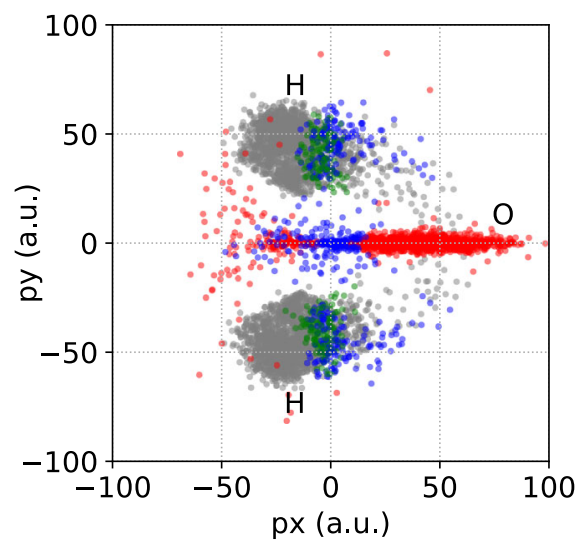

FIG. 10. Final ion momenta for $\mathrm{H}_{2} \mathrm{O}^{4+}$ in two different orientations. (a) The momenta are oriented such that the final oxygen momentum is oriented along the $x$ axis. (b) The momenta are oriented according to the initial geometry (oxygen is initially on the positive $x$ axis). Gray dots mark proton momenta; red dots mark oxygen momenta. Green dots mark proton momenta with a positive component along the corresponding oxygen momentum. Blue dots mark the corresponding partner proton and oxygen momenta.

the tail structure, i.e., the proton momenta that have contributions along the oxygen momentum (positive $x$ axis), with green color. The corresponding partner oxygen and proton momenta are marked with blue color. Figure 10(b) shows the same data, but here the momentum data are plotted in the molecular frame, i.e., where, in the initial geometry, the oxygen is oriented along the positive $x$ axis. The same color coding as in Fig. 10(a) is used. As can be seen, a qualitatively different picture arises in the two diagrams. One can clearly see that the blue and green momentum values concentrate in the region around $p_{x}=0$, i.e., where the molecule must have dissociated from an almost linear geometry. A substantial part of the tail structure must thus be associated with large $\mathrm{HOH}$ bending dynamics. Moreover, one can see that, in a number of trajectories, oxygen atoms are emitted in the negative $x$ axis direction, indicating that a substantial part of the trajectories shows overbending dynamics; i.e., the $\mathrm{HOH}$ bond angle increases beyond a linear geometry. From the simulations of the $\mathrm{H}_{2} \mathrm{O}^{2+}$ dynamics following single-photon absorption, we can quantify that after core ionization and Auger decay, $22 \%$ of the trajectories that dissociate in a three-bodyfragmentation channel show overbending.

\section{APPENDIX C: CORRELATION OF KINETIC ENERGY WITH DIFFERENT TIME DELAYS}

To obtain timing information, we exploit the fact that the KER is strongly correlated with the time between the two photoionization events $t_{P 2}-t_{P 1}$ [Fig. 4(a)]. In principle, the timing for the charging-up of the molecule and thereby the KER also depends on how fast Auger decays follow photoionization events. Figure 11 shows scatter plots of the kinetic energy release and various time-delay parameters: time between the two photoabsorptions, $t_{P 2}-t_{P 1}$ [Fig. 11(a)]; time between the first Auger decay and the first photoabsorption, $t_{A 1}-t_{P 1}$ [Fig. 11(b)]; and time between the second Auger decay and the second photoabsorption, $t_{A 2}-t_{P 2}$ [Fig. 11(c)]. The bottom plots [Figs. 11(d)-11(f)] show the corresponding histograms. As can be seen, the time between two photoionizations, $t_{P 2}-t_{P 1}$, shows a marked correlation with the resulting kinetic energy because the large delay times correspond to lower KER [see Fig. 11(a)]. The distribution of delay times $t_{P 2}-t_{P 1}$ [Fig. 11(d)] extends up to $50 \mathrm{fs}$, whereas large delay times are considerably less frequent. PPAA events particularly focus on short delay times, less than 5 fs. The time between the first photoionization and first Auger decay, $t_{A 1}-t_{P 1}$, shows almost no correlation with the KER [see Fig. 11(b)]; the values are distributed following an exponential decay, with a decay constant of about 3 fs [Fig. 11(e)]. For the PPAA events, no marked difference in this distribution can be seen. The time between the second photoionization and second Auger decay, $t_{A 2}-t_{P 2}$, shows a slight correlation to the KER for high kinetic energies [Fig. 11(c)]. The distribution of the values shown in Fig. 11(f) extends to somewhat larger values as compared to $t_{A 1}-t_{P 1}$ [Fig. 11(e)]; also here, 
(a)

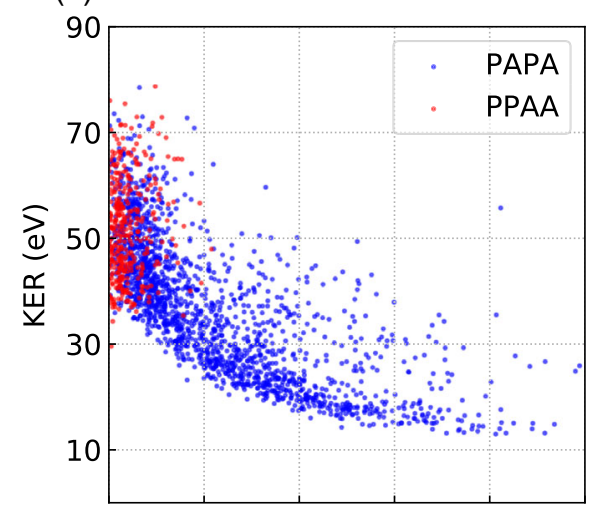

(d)

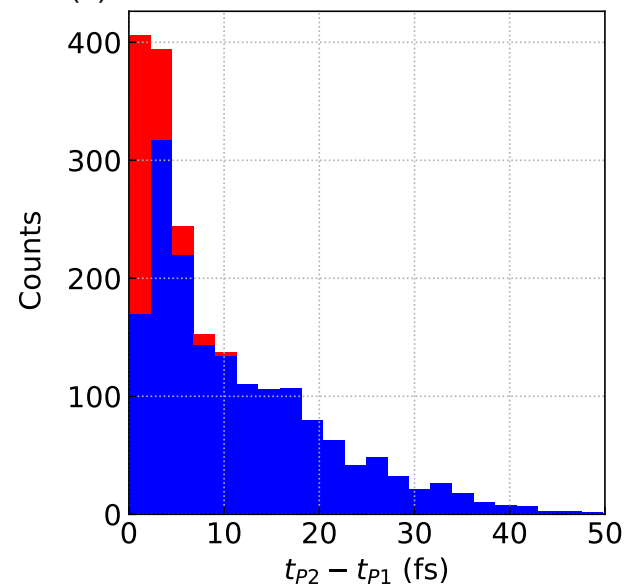

(b)

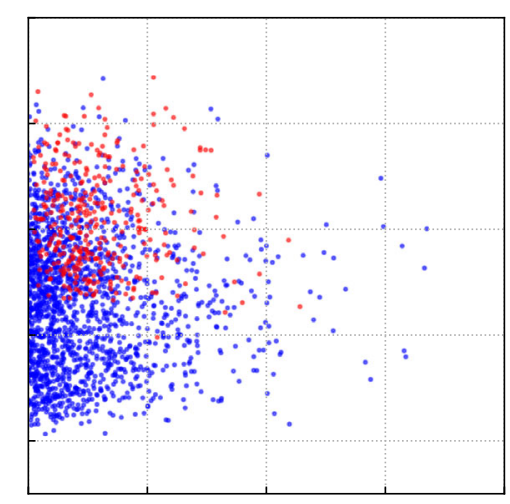

(e)

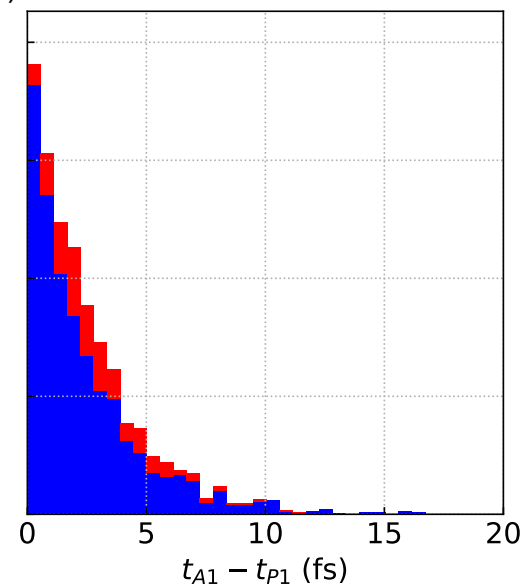

(c)

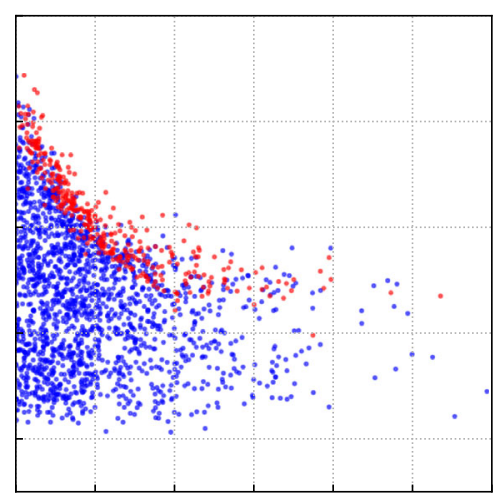

(f)

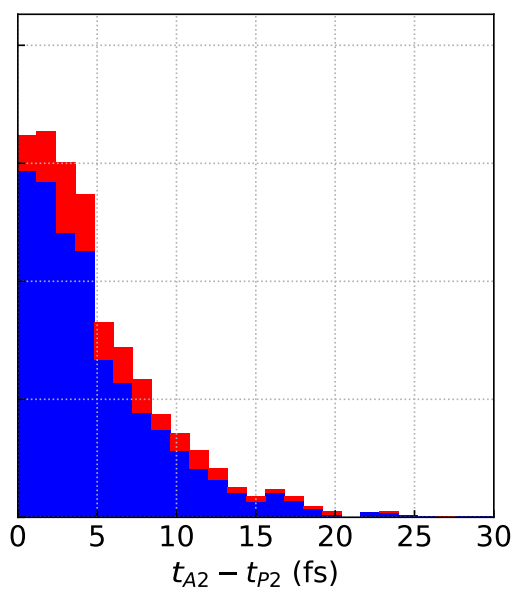

FIG. 11. Scatter plots [(a)-(c)] showing the relation of different ionization times with KER, and histograms [(d)-(f)] showing the distribution of ionization times for $\mathrm{H}_{2} \mathrm{O}$ and the coincidence with $\mathrm{O}^{2+}$ and $2 \mathrm{H}^{+}$. In the plots, $t_{P 1}$ and $t_{P 2}$ are the times of the two photoionization events, and $t_{A 1}$ and $t_{A 2}$ are the times of the two Auger decay steps. PAPA ionization sequences are marked in blue, and PPAA ionization sequences are marked in red.

the timing distribution of PPAA events is not markedly different as compared to PAPA events.

The dependence of the timing parameters on the KER is further quantified by Pearson correlation coefficients as $-0.73,0.01$, and -0.28 , for the correlation between KER and $t_{P 2}-t_{P 1}, t_{A 1}-t_{P 1}$, and $t_{A 2}-t_{P 2}$, respectively. We conclude that the time between two photoionization events, $t_{P 2}-t_{P 1}$, is the major factor determining the KER distribution.

\section{APPENDIX D: EXTENDED RESULTS FOR HIGHER CHARGE COINCIDENCES}

Figures 12 and 13 show the scatter plots (proton momentum vs proton momentum, proton angle vs proton angle) for the coincidence channels $\mathrm{O}^{4+}+2 \mathrm{H}^{+}$and $O^{6+}+2 \mathrm{H}^{+}$. The top row shows experimental data, and the bottom row shows data from the simulation. As can be seen, the features associated with strong unbending 

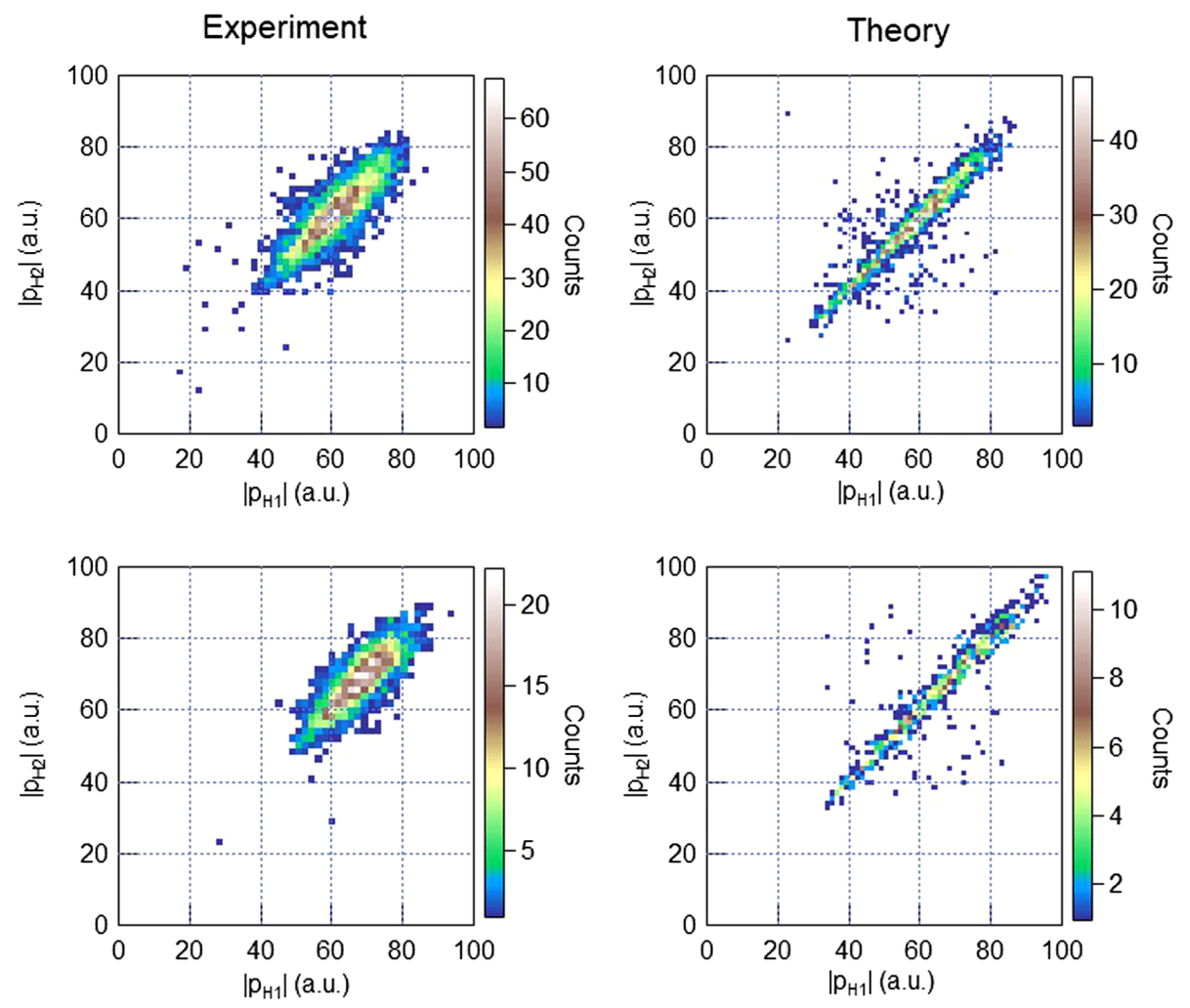

FIG. 12. Hydrogen absolute momenta pairs for different coincidence channels (top: 4-1-1; bottom: 6-1-1). The left column shows experimental results; the right column shows results from the simulation, respectively.

Experiment
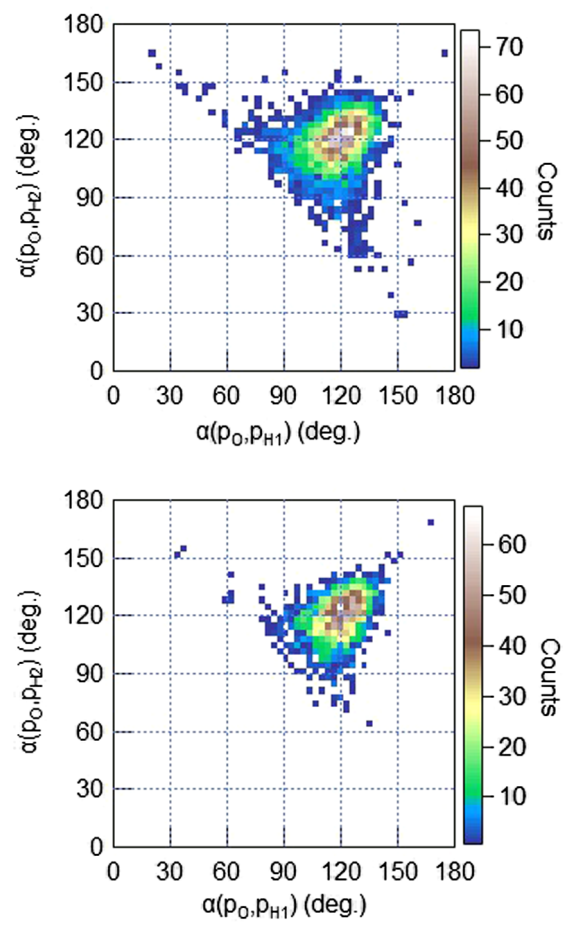

Theory
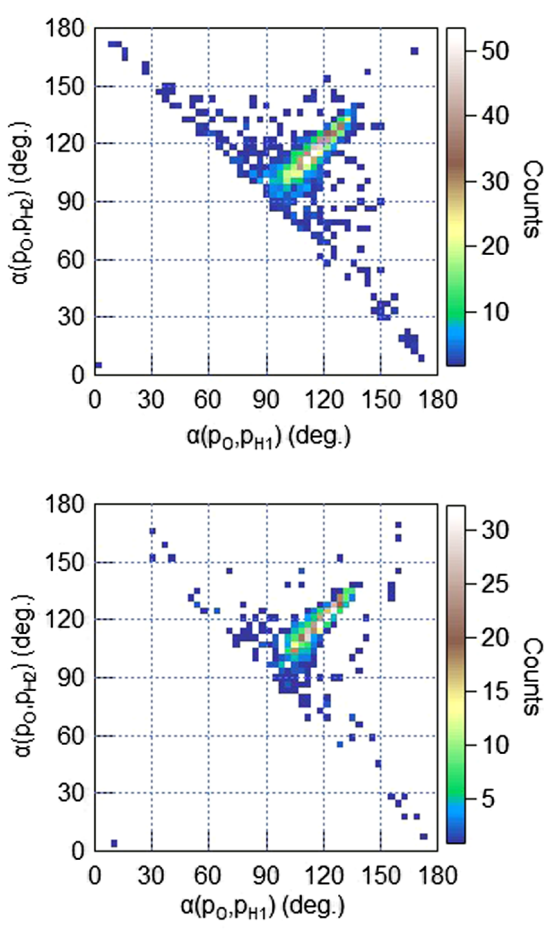

FIG. 13. Hydrogen-oxygen momentum angle pairs for different coincidence channels (top: 4-1-1; bottom: 6-1-1). The left column shows experimental results; the right column shows results from the simulation, respectively. 
dynamics and asymmetric dissociation become weaker at coincidences with higher oxygen charges.

\section{APPENDIX E: PULSE CALIBRATION}

To obtain a reliable value for the $\mathrm{x}$-ray fluence, we employed a calibration measurement with argon gas. The argon gas was exposed to the $\mathrm{x}$-ray pulse at identical experimental conditions as used for $\mathrm{H}_{2} \mathrm{O}$, and the ion yields for different charge states were recorded. To determine the spatial fluence distribution of the X-ray pulse, we compared the measured ion distribution with results from atomic calculations, employing different spatial fluence distributions. Figure 14 shows a comparison of calculated and measured ion yields. The red and blue lines show ion yields calculated with optimal parameters for single- and doubleGaussian spatial pulse shapes, fitted to experimental ion yields, as determined by the XCALIB tool [50]. A pulse duration of $30 \mathrm{fs}$ is assumed, and the charge states of +6 , +7 , and +16 are excluded for calibration, because of inaccuracies of calculated values $(+6$ and +7$)$ and plausible influence of the second harmonic contribution and pulse duration $(+16)$. As can be seen, the agreement is significantly improved when a double-Gaussian pulse shape is used. The resulting parameters that characterize the spatial pulse shape are as follows: the peak fluence of $7.0 \times 10^{11} \mathrm{ph} / \mu \mathrm{m}^{2}$ for a single Gaussian; and the peak fluence of $8.5 \times 10^{11} \mathrm{ph} / \mu \mathrm{m}^{2}$, the width ratio of 1.77 , and the fluence ratio of 0.355 when assuming a double Gaussian. Assuming a focal area of $1.6 \times 1.6 \mu \mathrm{m}^{2}$, the beamline transmission is calculated as $14 \%$, which is

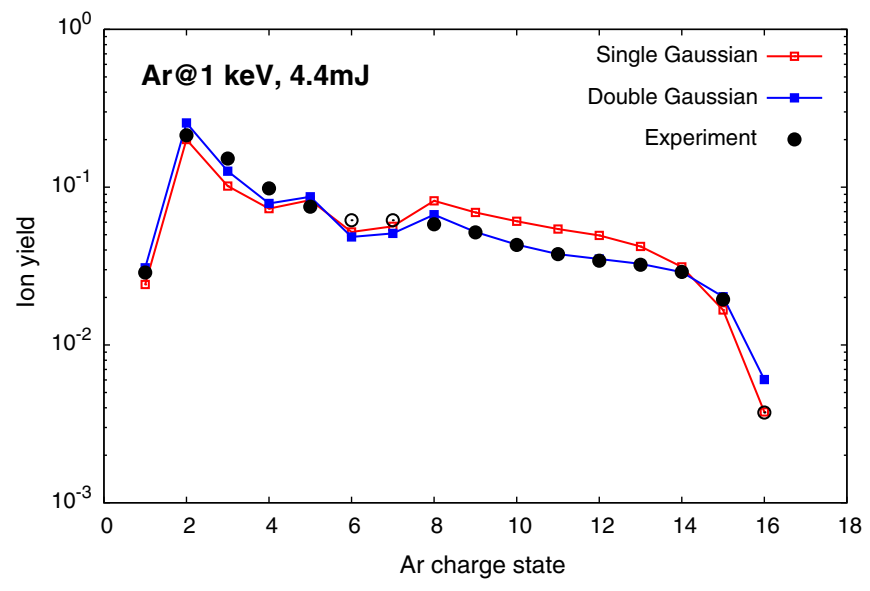

FIG. 14. Argon calibration measurement. The black circles show measured ion yields (open circles are excluded for calibration), and the lines show ion yields as calculated from atomic simulations. The red line shows results obtained with a single Gaussian for the spatial fluence distribution, while the blue line shows results obtained with a double-Gaussian fluence distribution. See text for the optimized parameters of the fluence distribution. equivalent to $45 \%$ with $2.9 \times 2.9 \mu \mathrm{m}^{2}$ since the calibration determines only the ratio between the transmission and the focal area. More details on the calibration procedure can be found in Ref. [50].

\section{APPENDIX F: FLUENCE DEPENDENCE}

The ionization and fragmentation dynamics of the molecule are linked to the respective timings of the photoionization events that depend, in principle, on the fluence value. Because the considered charge states $q$ can almost exclusively be associated with a respective number of photoabsorptions, it turns out that the fragmentation dynamics can be studied independently of the fluence. This can be understood based on the following arguments: Photoionization occurs predominantly at the oxygen core shell. Assuming that a created core hole is sufficiently rapidly refilled via Auger decay, the associated cross section can be considered to be constant throughout the whole ionization and fragmentation dynamics. Within such an approximation, the differential probability distribution for $n$ photoionization at times $t_{1}, \ldots t_{n}$ is given by

$$
\frac{d P^{(n)}\left(t_{1}, \ldots, t_{n}\right)}{d t_{1} \cdots d t_{n}}=(\sigma F)^{n} e^{-\sigma F} \prod_{m=1 \ldots n} f\left(t_{n}\right),
$$

where $\sigma$ is the photoionization cross section, $F$ is the fluence, and $f(t)$ is the temporal pulse profile. As can be seen, the fluence can be extracted into a timingindependent prefactor. As a consequence, the relative amount of, e.g., rapid ionization events with small $t_{2}-t_{1}$, compared to delayed ionization events with large $t_{2}-t_{1}$, is not affected by the fluence.

To verify the fluence independence of the studied ion momentum coincidence signal, we performed simulations for fluence values $10^{11} \mathrm{ph} / \mu \mathrm{m}^{2}, 2 \times 10^{11} \mathrm{ph} / \mu \mathrm{m}^{2}$, and $4 \times 10^{11} \mathrm{ph} / \mu \mathrm{m}^{2}$. These fluences cover the range that has relevant contributions to charge state +4 (see Fig. 7). Figure 15 shows the same analysis as in Figs. 4(a)-4(c) but for the three different fluence values. As suggested by Eq. (F1), the relative timing distribution and, consequently, the resulting fragmentation dynamics are barely affected by the fluence. Accordingly, the fractions for larger proton asymmetry and large proton angle are almost identical. We see, however, that the count rate of the coincidence signal for charge states +4 is affected, resulting in a reduced statistics, in particular, for the high fluence [Fig. 15(g)] because at this fluence ionization leading to charge states beyond +4 is more likely. The lower statistics results in slightly larger statistical error bars. Apart from some deviations within these error bars, the results at different fluence values are consistent, which confirms that when looking into particular ion coincidences, the fragmentation dynamics are barely affected by the fluence. 
(a)

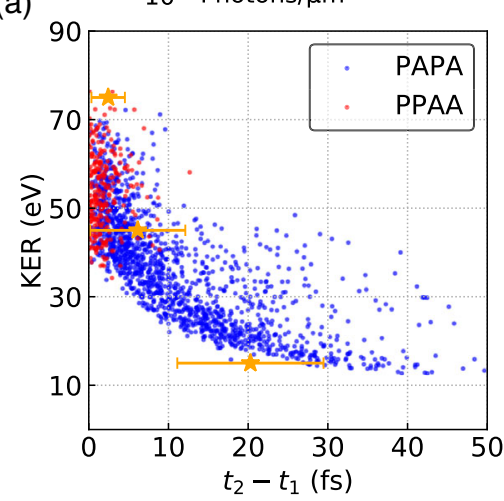

(d)

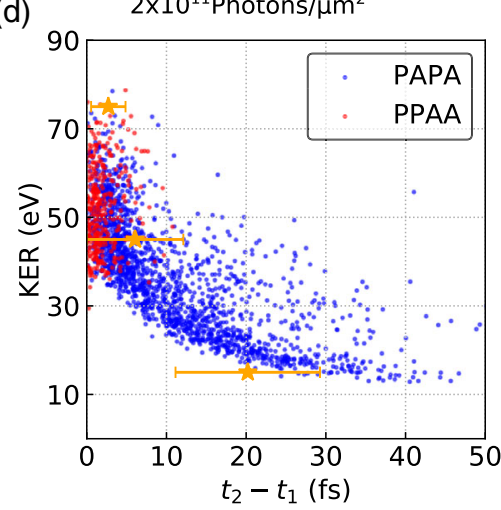

(g)

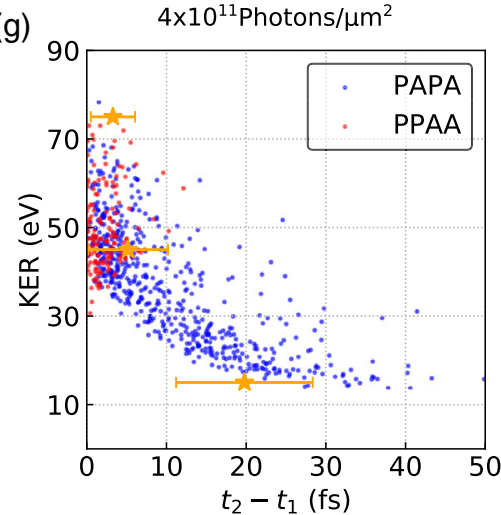

(b)

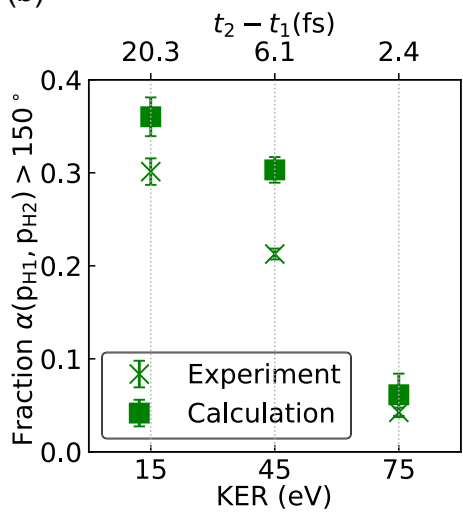

(e)

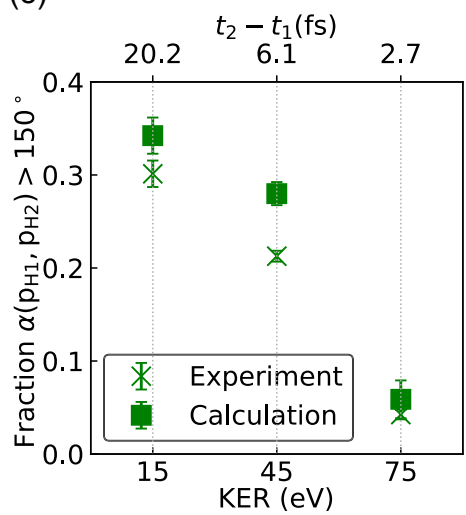

(h)

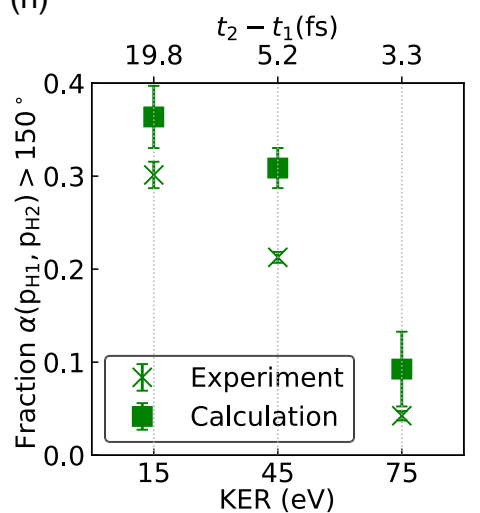

(c)

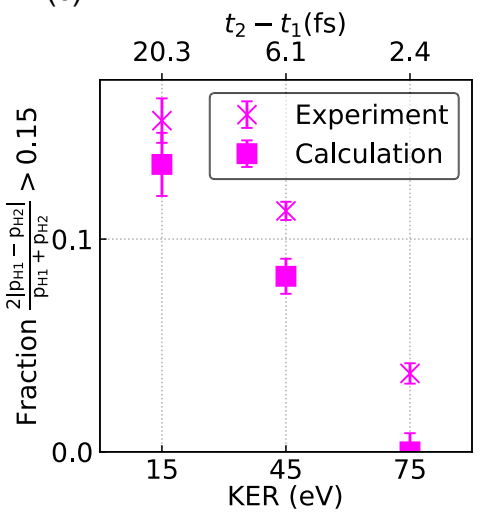

(f)

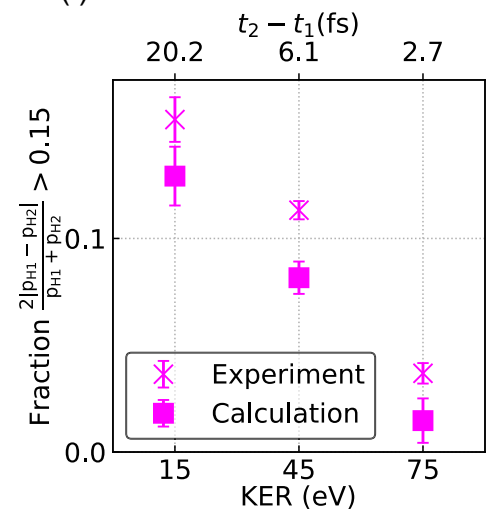

(i)

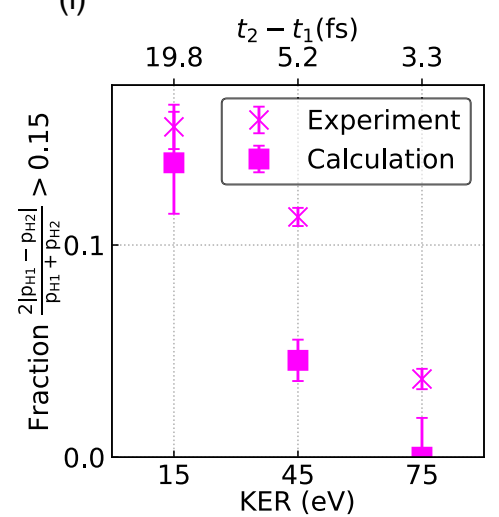

FIG. 15. Evolution in real time [same as Figs. 4(a)-(c) but for different fluences]. (a,d,g) KER as a function of time between the first and the second photoionization, $t_{2}-t_{1}$ (simulation), for different fluence values. The yellow stars mark average delay times for the three KER bins considered in (b), (e), (h) and (c), (f), (i). The bars indicate standard deviations of the delay times within the respective KER bins. (b,e,h) Ratio of coincidence events with large proton-proton momentum angle and large relative proton momentum asymmetry as a function of KER (experiment and simulation). (c,f,i) $\mathrm{HOH}$ bond angles and asymmetry ratio of the $\mathrm{OH}$ distances at the point in time of the second photoabsorption vs time delay with respect to the first photoabsorption (simulations) accompanied by selected snapshots of the molecular geometry. All plots are for coincidences with oxygen charge $2+$ and $2 \mathrm{H}^{+}$ions. The calculated results are obtained for different fluence values: (a)-(c) $10^{11} \mathrm{ph} / \mu \mathrm{m}^{2}$, (d)-(f) $2 \times 10^{11} \mathrm{ph} / \mu \mathrm{m}^{2}$, and (g)-(i) $4 \times 10^{11} \mathrm{ph} / \mu \mathrm{m}^{2}$.

We note that the relative contributions of the different ionization sequences PPAA vs PAPA are somewhat fluence dependent, as these dynamics are beyond the assumption of a constant cross section. For the three considered fluence values, we obtain a ratio of PPAA/PAPA of $0.18,0.21$, and
0.27 , respectively. A PPAA sequence, however, does not result in fragmentation dynamics that are significantly different from a PAPA sequence at small delay $t_{2}-t_{1}$ since both lead, more or less, to an almost immediate Coulomb explosion. 


\section{APPENDIX G: MAPPING OF KER TO TIME BETWEEN PHOTOIONIZATIONS FOR DIFFERENT PULSE SHAPES}

Figures 16(a)-16(c) show the KER as a function of time between photoionizations $t_{2}-t_{1}$ for the three different pulse shapes discussed in Fig. 9. Because the distribution of photoionization timings is considerably different for the different temporal pulses, the KER vs $t_{2}-t_{1}$ scatter plot also shows differences. However, all three distributions show a consistent trend. Regardless of the employed temporal pulse shape, a small delay time, $t_{2}-t_{1}<10 \mathrm{fs}$, can be associated with a KER $\geq 10 \mathrm{eV}$. A larger delay time, $t_{2}-t_{1}>20 \mathrm{fs}$, can be linked to a KER $<25 \mathrm{eV}$. Note that the 10-fs Gaussian pulse does not show any data for $t_{2}-t_{1}>20 \mathrm{fs}$. (a)

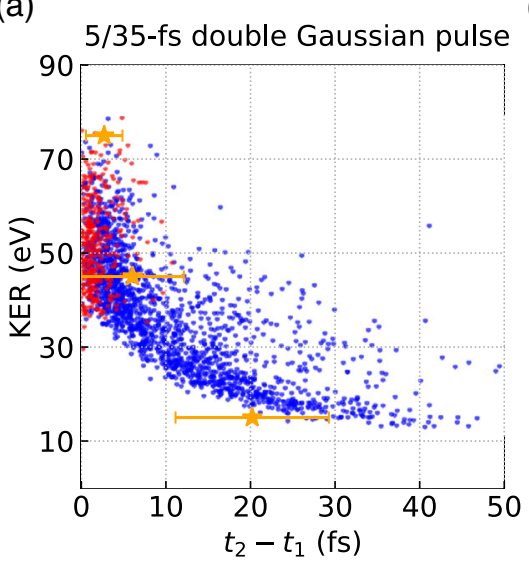

(d)

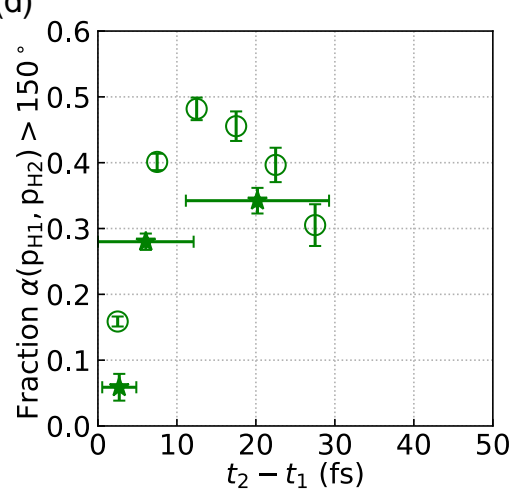

(g)

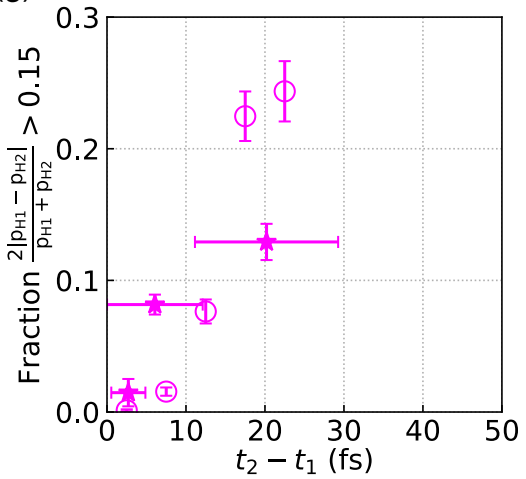

(b)

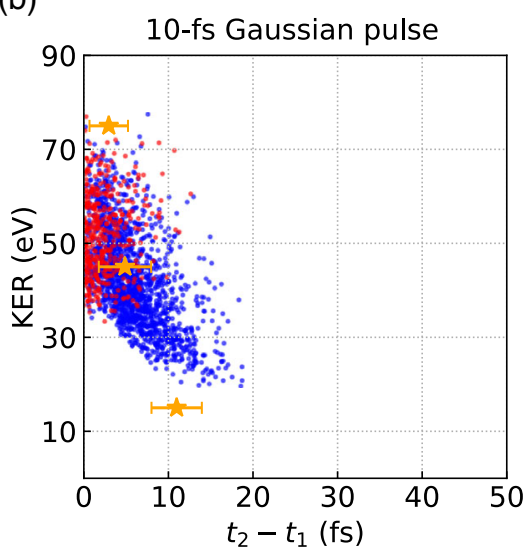

(e)

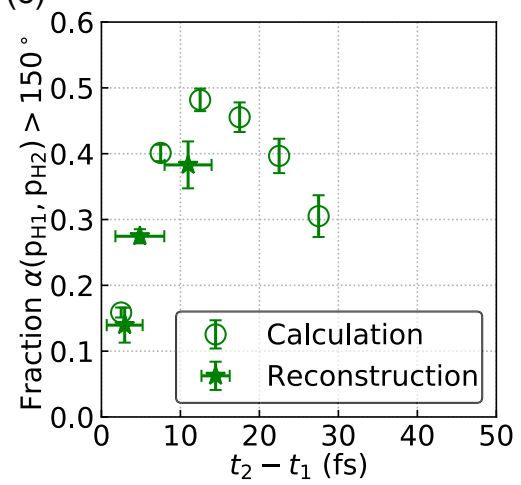

(h)

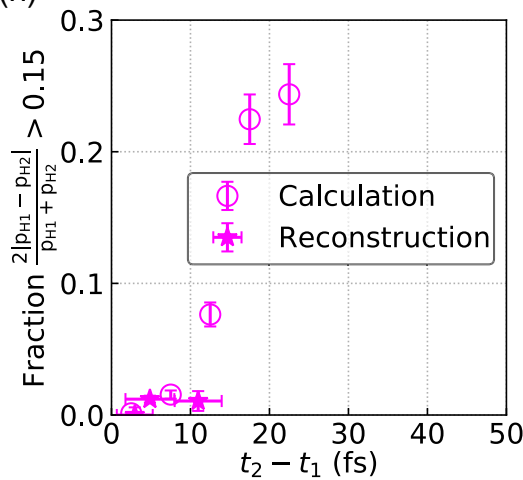

(c)

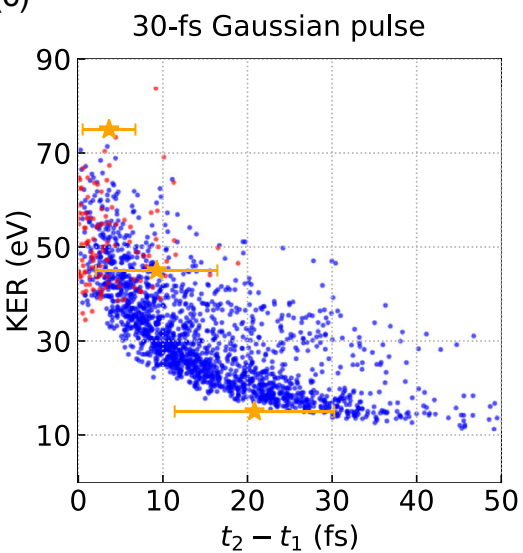

(f)

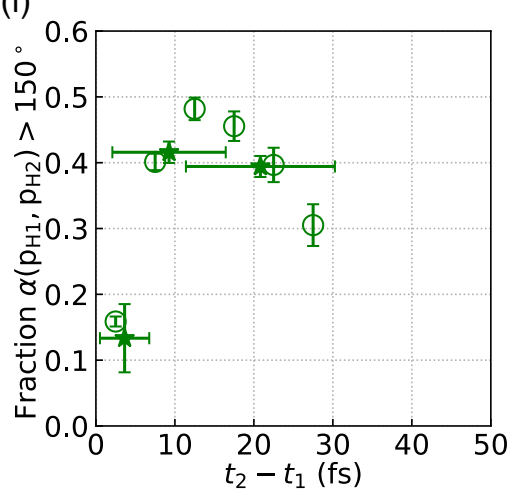

(i)

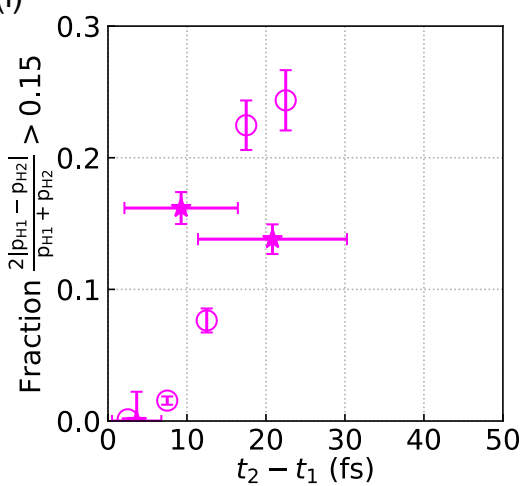

FIG. 16. (a)-(c) KER as a function of time between the first and the second photoionization $t_{2}-t_{1}$ for different pulse shapes. The yellow stars mark the average timing values for the selected KER bins. The error bar shows the standard deviation of $t_{2}-t_{1}$ within the bin. (d)-(f) Fraction of proton angles larger than $150^{\circ}$ as a function of $t_{2}-t_{1}$. (g)-(i) Fraction of proton asymmetry larger than 0.15 as a function of $t_{2}-t_{1}$. The circles show the data as extracted from the simulations, and the stars show data, where the timing information has been deduced by the KER-time relation. Vertical error bars indicate standard error, and horizontal error bars indicate the standard deviation of $t_{2}-t_{1}$ within the selected KER bin. 
For comparison, we employ the same KER bins as for the experimental data $(0-30 \mathrm{eV}, 30-60 \mathrm{eV}$, and $60-90 \mathrm{eV})$ to map timing information. However, because of the missing data for the 10-fs pulse, the lower KER bin indicates a too-small delay time of $t_{2}-t_{1}=11.0 \mathrm{fs}$ for this KER range. Furthermore, because of the somewhat shifted distribution, the delay time $t_{2}-t_{1}$ for the second KER bin is somewhat larger for the 30 -fs Gaussian pulse as compared to the 5/35-fs Gaussian pulse (9.3 fs instead of $6.1 \mathrm{fs})$. The remaining associations of KER with timing differ only slightly for the different pulse shapes.

To discuss the reliability of the extraction of timing information from the KER, we show in Figs. 16(d)-16(f) the fraction of final proton angles greater than $>150^{\circ}$ as a function of $t_{2}-t_{1}$ directly taken from the simulations. Because of the bending dynamics initiated by the first photoionization, this fraction increases rapidly and shows a maximum at $t_{2}-t_{1}=12 \mathrm{fs}$ and decreases for larger $t_{2}-t_{1}$. The stars show the reconstructed values, where the data have been binned according to the KER and assigned to a delay time $t_{2}-t_{1}$ corresponding to the average within the respective KER bin. The horizontal error bars indicate the standard deviation of $t_{2}-t_{1}$ within the KER bin. As can be seen, the trend indicating that larger $t_{2}-t_{1}$ leads to a larger proton angle within the first $10 \mathrm{fs}$ is qualitatively reproduced for all three employed temporal pulse shapes. The reconstruction of the data is clearly limited by the imprecise relation between KER and $t_{2}-t_{1}$, which is indicated by the broad distribution of delay times $t_{2}-t_{1}$ for a given KER range.

For the asymmetric stretching dynamics, the analogous comparison is shown in Figs. 16(g)-16(i). As one can see in the original simulation data shown by the open circles, the fraction of larger asymmetry in the proton momenta increases as a function of $t_{2}-t_{1}$ and reaches a value of about 0.22 at around $20 \mathrm{fs}$. For the double-Gaussian and the 30 -fs Gaussian pulse, the reconstructed data qualitatively reproduce the trend indicating larger asymmetry for larger delay time $t_{2}-t_{1}$. The reconstructed timings match within the indicated standard deviation for $t_{2}-t_{1}$ with the original simulation data. For the 10-fs Gaussian pulse, the asymmetry fraction remains close to zero, which is attributed to the fact that the pulse is too short to capture any asymmetric dynamics. Overall, the reconstruction of timing information for the asymmetry performs considerably worse compared to the one for the bending. The middle KER bins are associated with a too-small time delay (or a toolarge asymmetry fraction), whereas the lower KER bins (large $t_{2}-t_{1}$ ) show too little asymmetry as compared to the original simulation data. We link this discrepancy to the fact that the KER-time-delay dependence at larger $t_{2}-t_{1}$ is different for a two-body fragmentation compared to a threebody fragmentation. Thus, the KER-time-delay relation is expected to be biased because, for the same time delay, the two-body fragmentation resulting in the dissociation into
$\mathrm{OH}^{+}$and $\mathrm{H}^{+}$should result in a larger KER as compared to a three-body fragmentation into $\mathrm{O}+\mathrm{H}^{+}+\mathrm{H}^{+}$at the same delay. Consequently, the time delay $t_{2}-t_{1}$ for asymmetry in the middle KER bin is somewhat underestimated, and in the lowest KER bin, the fraction of asymmetry is also underestimated.

\section{APPENDIX H: IONIZATION TIMING STATISTICS}

In an alternative approach, inspection of higher charge coincidences allows us to infer timing information because they imply a larger number of absorbed photons and thus correlate with a shorter time between first and second photoionization. To elucidate this relation, here we inspect the distribution of time between the first and second photoionizations for a given number of absorbed photons. Assuming that the ionization cross section $\sigma(t)=\sigma$ is constant (by far, the dominant cross section is for core ionization, which is rapidly refilled via Auger decay), the differential probability to have a time $\tau_{2}=t_{2}-t_{1}$ between the first and the second photoionizations when there are in total $n$ total photoionizations is

$$
\begin{aligned}
\frac{d P^{(n)}\left(\tau_{2}\right)}{d \tau_{2}} & =(\sigma F)^{n} e^{-\sigma F} \int_{-\infty}^{\infty} d t_{1} f\left(t_{1}\right) f\left(t_{1}+\tau_{2}\right) \\
& \times \int_{0}^{\infty} d \tau_{3} \ldots d \tau_{n} \prod_{m=3 \ldots n} f\left(t_{1}+\tau_{2}+\sum_{k=3 \ldots m} \tau_{k}\right),
\end{aligned}
$$

where $\tau_{k}=t_{k}-t_{k-1}$ is the time between the $k$ th and $k-1$ th photoabsorptions, $f(t)$ is the temporal pulse profile, and $F$ the fluence [51]. We note that for very

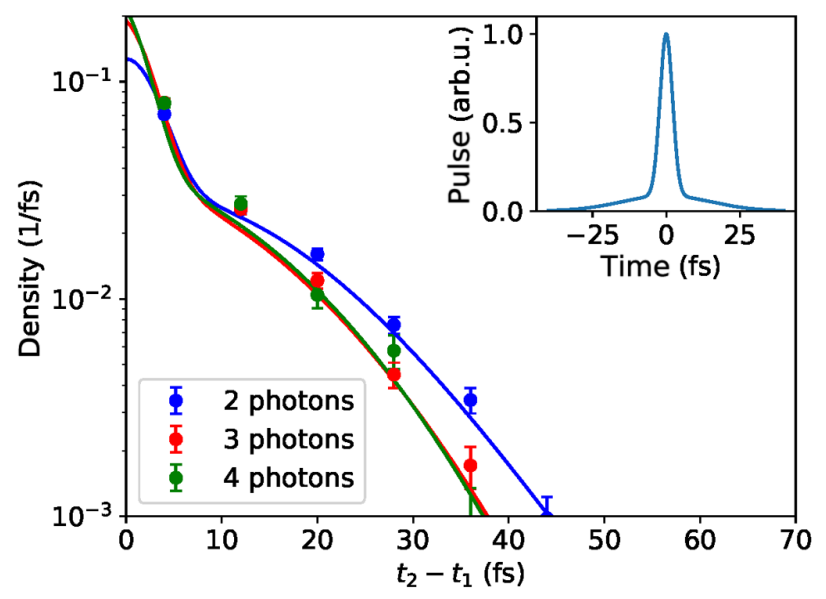

FIG. 17. Differential probability distribution of the time between the first and second photoionizations, $t_{2}-t_{1}$. The inset shows the employed double-Gaussian temporal pulse shape. Solid lines show results from an analytical model assuming constant ionization cross sections, and dots depict results from the actual simulation. Error bars depict standard errors. 
short time intervals $t_{2}-t_{1}$ close to or smaller than the corehole lifetime of about $3 \mathrm{fs}$ [21], the analytical model in Eq. (G1) is not applicable, as a consequence of the finite core-hole lifetime that causes a transiently decreased photoionization cross section [52,53].

Figure 17 shows the resulting photoionization timing distributions. The solid lines are due to the analytical model [Eq. (G1)], and the points are from the simulation data. As one might intuitively expect, the probability to have short times $t_{2}-t_{1}$ is larger when more photons are absorbed within the whole pulse. For the temporal pulse shape used here, one can see that the three probability curves for two-, three-, and four-photon absorption events cross at a delay time $t_{2}-t_{1}$ of about $8 \mathrm{fs}$. This indicates that the probability for having a delay time $t_{2}-t_{1}>8 \mathrm{fs}$ is relatively suppressed when we inspect higher charge coincidences that result from a larger number of absorbed photons. At the same time, for lower charge coincidences, it is more likely to have a delay time $t_{2}-t_{1}<8$ fs since lower charges imply fewer absorbed photons. Since the dynamical momentum patterns discussed in the main text are predominantly a result of the dynamics between the first and second photoabsorptions, the suppression of these patterns at coincidences with higher charges indicates that the underlying dynamics occur on a timescale smaller than about 8 fs.

[1] B. Boudaïffa, P. Cloutier, D. Hunting, M. A. Huels, and L. Sanche, Resonant Formation of DNA Strand Breaks by Low-Energy (3 to $20 \mathrm{eV}$ ) Electrons, Science 287, 1658 (2000).

[2] M. Baumann, M. Krause, J. Overgaard, J. Debus, S. M. Bentzen, J. Daartz, C. Richter, D. Zips, and T. Bortfeld, Radiation Oncology in the Era of Precision Medicine, Nat. Rev. Cancer 16, 234 (2016).

[3] B. C. Garrett et al., Role of Water in Electron-Initiated Processes and Radical Chemistry: Issues and Scientific Advances, Chem. Rev. 105, 355 (2005).

[4] R. W. Carlson et al., Hydrogen Peroxide on the Surface of Europa, Science 283, 2062 (1999).

[5] M. Blanc, D. J. Andrews, A. J. Coates, D. C. Hamilton, C. M. Jackman, X. Jia, A. Kotova, M. Morooka, H. T. Smith, and J.H. Westlake, Saturn Plasma Sources and Associated Transport Processes, Space Sci. Rev. 192, 237 (2015).

[6] I. G. Draganić, Radiolysis of Water: A Look at Its Origin and Occurrence in the Nature, Radiat. Phys. Chem. 72, 181 (2005).

[7] A. Sankari, C. Stråhlman, R. Sankari, L. Partanen, J. Laksman, J. A. Kettunen, I. F. Galván, R. Lindh, P.-Å. Malmqvist, and S. L. Sorensen, Non-Radiative Decay and Fragmentation in Water Molecules after $1 a_{1}^{-1} 4 a_{1}$ Excitation and Core Ionization Studied by Electron-EnergyResolved Electron-Ion Coincidence Spectroscopy, J. Chem. Phys. 152, 074302 (2020).
[8] Z.-H. Loh et al., Observation of the Fastest Chemical Processes in the Radiolysis of Water, Science 367, 179 (2020).

[9] E. Kamarchik, O. Kostko, J. M. Bowman, M. Ahmed, and A. I. Krylov, Spectroscopic Signatures of Proton Transfer Dynamics in the Water Dimer Cation, J. Chem. Phys. 132, 194311 (2010).

[10] O. Marsalek, C. G. Elles, P. A. Pieniazek, E. Pluharova, J. VandeVondele, S. E. Bradforth, and P. Jungwirth, Chasing Charge Localization and Chemical Reactivity Following Photoionization in Liquid Water, J. Chem. Phys. 135, 224510 (2011).

[11] O. Fuchs et al., Isotope and Temperature Effects in Liquid Water Probed by X-Ray Absorption and Resonant X-Ray Emission Spectroscopy, Phys. Rev. Lett. 100, 027801 (2008).

[12] T. Tokushima, Y. Harada, O Takahashi, Y. Senba, H. Ohashi, L. G. M. Pettersson, A. Nilsson, and S. Shin, High Resolution X-Ray Emission Spectroscopy of Liquid Water: The Observation of Two Structural Motifs, Chem. Phys. Lett. 460, 387 (2008).

[13] S. Thürmer, M. Oncak, N. Ottosson, R. Seidel, U. Hergenhahn, S. E. Bradforth, P. Slavicek, and B. Winter, On the Nature and Origin of Dicationic, Charge-Separated Species Formed in Liquid Water on X-Ray Irradiation, Nat. Chem. 5, 590 (2013).

[14] M. N. Piancastelli, A. Hempelmann, F. Heiser, O. Gessner, A. Rüdel, and U. Becker, Resonant Photofragmentation of Water at the Oxygen K Edge by High-Resolution Ion-Yield Spectroscopy, Phys. Rev. A 59, 300 (1999).

[15] J. H. Sanderson, A. El-Zein, W. A. Bryan, W. R. Newell, A. J. Langley, and P. F. Taday, Geometry Modifications and Alignment of $\mathrm{H}_{2} \mathrm{O}$ in an Intense Femtosecond Laser Pulse, Phys. Rev. A 59, R2567 (1999).

[16] F. Légaré, K. F. Lee, V. Litvinyuk, P. W. Dooley, S. S. Wesolowski, P. R. Bunker, P. Dombi, F. Krausz, A. D. Bandrauk, D. M. Villeneuve, and P. B. Corkum, Laser Coulomb-Explosion Imaging of Small Molecules, Phys. Rev. A 71, 013415 (2005).

[17] T. J. Van Huis, S. S. Wesolowski, Y. Yamaguchi, and H. F. Schaefer, Scratching the Surface of the Water Dication, J. Chem. Phys. 110, 11856 (1999).

[18] B. Gervais, E. Giglio, L. Adoui, A. Cassimi, D. Duflot, and M.E. Galassi, The $\mathrm{H}_{2} \mathrm{O}^{2+}$ Potential Energy Surfaces Dissociating into $\mathrm{H}^{+} / \mathrm{OH}^{+}$: Theoretical Analysis of the Isotopic Effect, J. Chem. Phys. 131, 024302 (2009).

[19] Z. L. Streeter, F. L. Yip, R. R. Lucchese, B. Gervais, T. N. Rescigno, and C.W. McCurdy, Dissociation Dynamics of the Water Dication Following One-Photon Double Ionization. I. Theory, Phys. Rev. A 98, 053429 (2018).

[20] D. Reedy et al., Dissociation Dynamics of the Water Dication Following One-Photon Double Ionization. II. Experiment, Phys. Rev. A 98, 053430 (2018).

[21] L. Inhester, C. F. Burmeister, G. Groenhof, and H. Grubmüller, Auger Spectrum of a Water Molecule after Single and Double Core Ionization, J. Chem. Phys. 136, 144304 (2012).

[22] L. Inhester, K. Hanasaki, Y. Hao, S.-K. Son, and R. Santra, $X$-Ray Multiphoton Ionization Dynamics of a Water 
Molecule Irradiated by an X-Ray Free-Electron Laser Pulse, Phys. Rev. A 94, 023422 (2016).

[23] Y. Hao, L. Inhester, K. Hanasaki, S.-K. Son, and R. Santra, Efficient Electronic Structure Calculation for Molecular Ionization Dynamics at High X-Ray Intensity, Struct. Dyn. 2, 041707 (2015).

[24] J. M. Schäfer, L. Inhester, S.-K. Son, R. F. Fink, and R. Santra, Electron and Fluorescence Spectra of a Water Molecule Irradiated by an X-Ray Free-Electron Laser Pulse, Phys. Rev. A 97, 053415 (2018).

[25] X. Li, L. Inhester, T. Osipov, R. Boll, R. Coffee, J. Cryan, A. Gatton, T. Gorkhover, G. Hartman, M. Ilchen, A. Knie, M.-F. Lin, M. P. Minitti, C. Weninger, T. J. A. Wolf, S.-K. Son, R. Santra, D. Rolles, A. Rudenko, and P. Walter, Electron-Ion Coincidence Measurements of Molecular Dynamics with Intense X-Ray Pulses, Sci. Rep. 11, 505 (2021).

[26] H. Niikura, F. Légaré, R. Hasbani, A. D. Bandrauk, M. Yu. Ivanov, D. M. Villeneuve, and P. B. Corkum, Sub-LaserCycle Electron Pulses for Probing Molecular Dynamics, Nature (London) 417, 917 (2002).

[27] F. Légaré, I. V. Litvinyuk, P. W. Dooley, F. Quéré, A. D. Bandrauk, D. M. Villeneuve, and P. B. Corkum, TimeResolved Double Ionization with Few Cycle Laser Pulses, Phys. Rev. Lett. 91, 093002 (2003).

[28] D. Winkoun, G. Dujardin, L. Hellner, and M. J. Besnard, One- and Two-Step Double Photoionisation Processes in Valence Shells of $\mathrm{H}_{2} \mathrm{O}$, J. Phys. B 21, 1385 (1988).

[29] K. H. Tan, C. E. Brion, Ph. E. van der Leeuw, and M. J. van der Wiel, Absolute Oscillator Strengths (10-60 eV) for the Photoabsorption, Photoionisation and Fragmentation of $\mathrm{H}_{20}$ s, Chem. Phys. 29, 299 (1978).

[30] H. B. Pedersen et al., Photolysis of Water-Radical Ions $\mathrm{H}_{2} \mathrm{O}^{+}$in the XUV: Fragmentation through Dicationic States, Phys. Rev. A 87, 013402 (2013).

[31] H. Siegbahn, L. Asplund, and P. Kelfve, The Auger Electron Spectrum of Water Vapour, Chem. Phys. Lett. 35, 330 (1975).

[32] L. J. Frasinski et al., Dynamics of Hollow Atom Formation in Intense X-Ray Pulses Probed by Partial Covariance Mapping, Phys. Rev. Lett. 111, 073002 (2013), and references therein.

[33] L. Fang et al., Multiphoton Ionization as a Clock to Reveal Molecular Dynamics with Intense Short X-Ray Free Electron Laser Pulses, Phys. Rev. Lett. 109, 263001 (2012).

[34] See Supplemental Material at http://link.aps.org/ supplemental/10.1103/PhysRevX.11.041044 for a series of movies in which the simulated dynamics of the $\mathrm{H}_{2} \mathrm{O}$ molecule after one-photon absorption can be visualized.

[35] A. Barty, J. Küpper, and H. N. Chapman, Molecular Imaging Using X-Ray Free-Electron Lasers, Annu. Rev. Phys. Chem. 64, 415 (2013).

[36] A. H. Zewail, 4D Ultrafast Electron Diffraction, Crystallography, and Microscopy, Annu. Rev. Phys. Chem. 57, 65 (2006).

[37] https://in.xfel.eu/metadata/doi/10.22003/XFEL.EU-DATA002150-00
[38] W. Decking et al., A MHz-Repetition-Rate Hard X-Ray Free-Electron Laser Driven by a Superconducting Linear Accelerator, Nat. Photonics 14, 391 (2020).

[39] T. Tschentscher, C. Bressler, J. Grünert, A. Madsen, A. P. Mancuso, M. Meyer, A. Scherz, H. Sinn, and U. Zastrau, Photon Beam Transport and Scientific Instruments at the European XFEL, Appl. Sci. 7, 592 (2017).

[40] J. Ullrich, R. Moshammer, A. Dorn, R. Dörner, L. Ph. H. Schmidt, and H. Schmidt-Böcking, Recoil-Ion and Electron Momentum Spectroscopy: Reaction-Microscopes, Rep. Prog. Phys. 66, 1463 (2003).

[41] O. Jagutzki, J. S. Lapington, L. B. C. Worth, U. Spillman, V. Mergel, and H. Schmidt-Böcking, Position Sensitive Anodes for MCP Read-out Using Induced Charge Measurement, Nucl. Instrum. Methods Phys. Res., Sect. A 477, 256 (2002).

[42] P. C. Hariharan and J. A. Pople, The Influence of Polarization Functions on Molecular Orbital Hydrogenation Energies, Theor. Chim. Acta 28, 213 (1973).

[43] A. Rudenko et al., Femtosecond Response of Polyatomic Molecules to Ultra-Intense Hard X-Rays, Nature (London) 546, 129 (2017).

[44] P. J. Richardson, J. H. D. Eland, P. G. Fournier, and D. L. Cooper, Spectrum and Decay of the Doubly Charged Water Ion, J. Chem. Phys. 84, 3189 (1986).

[45] S. Legendre, E. Giglio, M. Tarisien, A. Cassimi, B. Gervais, and L. Adoui, Isotopic Effects in Water Dication Fragmentation, J. Phys. B 38, L233 (2005).

[46] N. Neumann et al., Fragmentation Dynamics of $\mathrm{CO}_{2}^{3+}$ Investigated by Multiple Electron Capture in Collisions with Slow Highly Charged Ions, Phys. Rev. Lett. 104, 103201 (2010).

[47] C. Wu et al., Nonsequential and Sequential Fragmentation of $\mathrm{CO}_{2}^{3+}$ in Intense Laser Fields, Phys. Rev. Lett. 110, 103601 (2013).

[48] B. Wales et al., Coulomb Imaging of the Concerted and Stepwise Break up Processes of OCS Ions in Intense Femtosecond Laser Radiation, J. Electron Spectrosc. Relat. Phenom. 195, 332 (2014).

[49] R. Guillemin et al., Selecting Core-Hole Localization or Delocalization in $\mathrm{CS}_{2}$ by Photofragmentation Dynamics, Nat. Commun. 6, 6166 (2015).

[50] K. Toyota, Z. Jurek, S.-K. Son, H. Fukuzawa, K. Ueda, N. Berrah, B. Rudek, D. Rolles, A. Rudenko, and R. Santra, xcalib: A Focal Spot Calibrator for Intense X-Ray Free-Electron Laser Pulses Based on the Charge State Distributions of Light Atoms, J. Synchrotron Radiat. 26, 1017 (2019).

[51] X. Li, R. Boll, D. Rolles, and A. Rudenko, Simple Model for Sequential Multiphoton Ionization by Ultraintense X Rays, Phys. Rev. A 104, 033115 (2021).

[52] M. Hoener et al., Ultraintense X-Ray Induced Ionization, Dissociation, and Frustrated Absorption in Molecular Nitrogen, Phys. Rev. Lett. 104, 253002 (2010).

[53] L. Young et al., Femtosecond Electronic Response of Atoms to Ultra-Intense X-Rays, Nature (London) 466, 56 (2010). 